Research Article

\title{
Glycolysis-Related Genes Serve as Potential Prognostic Biomarkers in Clear Cell Renal Cell Carcinoma
}

\author{
Yan Zhang, ${ }^{1,2}$ Mingying Chen, ${ }^{1}$ Meihong Liu, ${ }^{3}$ Yingkun Xu $\mathbb{D}^{4},{ }^{4}$ and Guangzhen $W u\left(\mathbb{D}{ }^{5}\right.$ \\ ${ }^{1}$ Department of Clinical Laboratory, The First Affiliated Hospital of Dalian Medical University, Dalian, Liaoning 116011, China \\ ${ }^{2}$ Department of Clinical Laboratory, The First People's Hospital of Linhai, Taizhou, Zhejiang 317000, China \\ ${ }^{3}$ Department of Respiratory, The First Affiliated Hospital of Dalian Medical University, Dalian, Liaoning 116011, China \\ ${ }^{4}$ Department of Urology, Shandong Provincial Hospital, Cheeloo College of Medicine, Shandong University, Jinan, \\ Shandong 250021, China \\ ${ }^{5}$ Department of Urology, The First Affiliated Hospital of Dalian Medical University, Dalian, Liaoning 116011, China
}

Correspondence should be addressed to Yingkun Xu; yingkunxu@hotmail.com and Guangzhen Wu; wuguang0613@hotmail.com

Received 18 November 2020; Revised 1 January 2021; Accepted 5 January 2021; Published 25 January 2021

Academic Editor: Shahnawaz Rehman

Copyright (c) 2021 Yan Zhang et al. This is an open access article distributed under the Creative Commons Attribution License, which permits unrestricted use, distribution, and reproduction in any medium, provided the original work is properly cited.

\begin{abstract}
Metabolic rearrangement is a marker of cancer that has been widely studied in recent years. One of the major metabolic characteristics of tumor cells is the high levels of glycolysis, even under aerobic conditions, a phenomenon that is called the "Warburg effect." We investigated the expression and copy number variation (CNV) frequency of all glycolysis-related genes in multiple cancer types and found many differentially expressed genes, particularly in clear cell renal cell carcinoma (ccRCC). Single nucleotide variants (SNVs) showed that the overall average mutation frequency for all genes was low. The purpose of this study was to establish a predictive model by studying glycolysis-related genes in ccRCC. We compared the expression of glycolysis-related genes in 539 ccRCC tissues and 72 normal renal tissues from The Cancer Genome Atlas dataset and identified 17 upregulated and 26 downregulated genes. Pathway analysis revealed that PSAT1 and SDHB could activate the cell cycle, RPIA could activate the DNA damage response, and HK3 could activate apoptosis and EMT signaling, while PDK2 could inhibit apoptosis. The results of the drug sensitivity analysis suggested that some of these differentially expressed genes were positively correlated with drug sensitivity. Thirteen genes were selected from the gene coexpression network and the LASSO regression analysis. The Kaplan-Meier overall survival curves showed that the expression of upregulated genes in ccRCC patients was associated with lower overall survival. We established a predictive model consisting of 13 genes (RPIA, G6PD, PSAT1, ENO2, HK3, IDH1, PDK4, PGM2, PGK1, FBP1, OGDH, SUCLA2, and SUCLG2). This predictive model correlated well with the development and progression of ccRCC. Thus, it is of great value in the diagnosis and prognostic evaluation of ccRCC and may aid the identification of potential prognostic biomarkers and drug targets.
\end{abstract}

\section{Introduction}

Renal cell carcinoma (RCC) is the third most common malignant cancer of the urinary system and accounts for approximately $90 \%$ of all malignant renal tumors $[1,2]$. According to recent statistics, more than 400,000 new cases of RCC have been reported, resulting in approximately 175,000 deaths worldwide annually [3]. In the United States alone, 73,820 new cases of RCC are reported each year and 14,770 people die from this disease [4]. Clear cell renal cell carcinoma (ccRCC) is the most common and fatal subtype, accounting for $75 \%$ of all RCCs [5]. As ccRCC is prone to metastasis in the early stages, approximately one-third of the patients exhibit distant metastases at the time of diagnosis [6]. These patients may have missed the best window for treatment. In addition, ccRCC is resistant to radiotherapy and traditional chemotherapy and has a poor prognosis.

For ccRCC, the gold standards for clinical diagnosis include computerized tomography (CT) and histopathological analyses. During the follow-up, CT is routinely performed to gauge disease progression, but histopathological analysis is an invasive procedure, which is not easily accepted by 
patients and is not suitable for regular monitoring. Moreover, it is difficult to detect small pathological changes in the early stages using CT scans, and there is a risk of radiation damage in patients who need long-term monitoring. At present, the identification of novel diagnostic and prognostic markers for ccRCC is a necessity.

In the early 1920s, Otto Warburg found that even with sufficient oxygen supply, cancer cells converted glucose into lactate [7]. Later, researchers termed this phenomenon as "aerobic glycolysis" or the "Warburg effect" [8]. The Warburg effect is frequently observed in tumors; it is one of the key mechanisms underlying carcinogenesis, and many studies have investigated tumor-related glycolytic genes in recent years $[9,10]$. To date, the Warburg effect and the glycolytic genes have been studied in lung cancer, breast cancer, ovarian cancer, gastric cancer, liver cancer, colorectal cancer, esophageal cancer, prostate cancer, bladder cancer, and other tumors [11-19], but there are few reports on ccRCC.

We used bioinformatics to explore glycolytic genes that are differentially expressed in 539 ccRCC tissues and 72 normal control tissues from The Cancer Genome Atlas (TCGA). A predictive model containing 13 genes was established after performing a series of investigations, including pathway analysis, drug sensitivity analysis, gene coexpression analysis, Lasso regression analysis, and survival curve analysis. This model showed great promise for the diagnosis and prognosis of ccRCC and may help in the identification of potential prognostic biomarkers and drug targets in ccRCC.

\section{Materials and Methods}

2.1. Data Collection. We acquired the data, including CNV, $\mathrm{SNV}$, and mRNA expression for ccRCC patients, from The Cancer Genome Atlas Database (TCGA) (https:// cancergenome.nih.gov/) [20]. TCGA dataset contained 539 ccRCC tissue samples and 72 normal control samples. In addition, we screened 53 glycolysis-related genes based on a literature survey [21].

2.2. Pathway Analysis. We analyzed the activation and inhibition of pathways using GSCALite (http://bioinfo.life.hust .edu.cn/web/GSCALite/) [22], which enables the analysis of the following: differential expression between tumor and normal tissues, survival between tumor and normal tissues, pathways related to gene expression, miRNA gene regulatory networks, and drug sensitivity.

2.3. Gene Coexpression Network and Transcriptional Factor Network. The gene coexpression network of glycolysisrelated genes and heat maps of transcriptional factors were generated using $\mathrm{R}$. We used the Corrplot software package for gene coexpression analysis and the Gene Expression Profiling Interactive Analysis (GEPIA) (http://gepia.cancer-pku $. \mathrm{cn} /$ ) [23] for analyzing the correlation between different genes. The overall survival (OS) and disease-free survival (DFS) of ccRCC patients were collected from the GEPIA website. Cytoscape was used to visualize the networks of coexpressed genes and transcriptional factors and identified differentially expressed genes [24].
2.4. Data Processing and Analysis. We downloaded the official R software from the CRAN website (https://www.rproject.org/). As the $\mathrm{R}$ software is complex to navigate, we used RStudio, which is a simple and powerful operation platform (https://www.rstudio.com/). In terms of data processing and analysis, Perl and other $\mathrm{R}$ packages were used in this study. The heat maps were generated using Phatmap. The Limma software package was used to perform differential expression analysis of glycolysis-related genes and transcriptional factors. Glmnet and Survival software packages were used to perform the LASSO regression analysis. The survival curves were generated using the Survival software package, and the ROC curves were analyzed and drawn using the survival ROC software package. A $p<0.05$ indicated a significant difference between the two groups of data.

\section{Results}

3.1. Identification of Glycolysis-Related Genes in ccRCC. We searched the literature and identified 53 genes involved in glycolysis. We used these genes to map the glycolysis and tricarboxylic acid cycle pathways (Figure 1). First, we investigated the alteration in the expression of the glycolysisrelated genes in 14 cancer types. The results revealed the presence of many differentially expressed glycolysis-related genes in various cancers compared to that in the control samples (Figure 2(a)). We then explored the copy number variation $(\mathrm{CNV})$ alteration frequency for all genes and found that $\mathrm{CNV}$ alterations were widespread. The genes with $\mathrm{CNV}$ amplifications exhibited a significantly higher expression in cancer tissues than in normal controls (e.g., HK3 and ENO2), while the genes with CNV deletions exhibited significantly lower expression (e.g., ALDOB and PSAT1) (Figure 2(b)). Single nucleotide variants (SNVs) showed that the overall average mutation frequency of all genes was low, especially in ccRCC (0.00-1.09\%; Figure 2(c)). Combined with the changes in gene expression, we speculated that $\mathrm{CNV}$ alterations may be one of the important mechanisms for modifying gene expression in ccRCC.

We first explored the relationship between gene expression levels and the hazard ratio (HR) of ccRCC. The OS and disease-free survival (DFS) of ccRCC patients were obtained from the GEPIA website. The high expression of HK3, ENO2, ENO3, and PSAT1 indicated a short OS and a high risk of developing renal cancer. The high expression of GPI, FBP1, ALDOB, and SUCLA2 indicated longer OS and a low risk of developing renal cancer (Figure 3(a)). The high expression of GAPDH, PSAT1, PGLS, and LDHC indicated a short DFS and a high risk of developing renal cancer. The high expression of FBP1, ALDOB, LDHD, and SUCLA2 indicated a longer DFS and a low risk of developing renal cancer (Figure 3(b)). Next, we compared the expression levels in 539 ccRCC tissues and 72 normal renal tissues in TCGA dataset and obtained 43 differentially expressed glycolysis-related genes. Compared with those in the normal control group, patients with ccRCC exhibited higher expression of PGK1, GPI, ALDOA, TPI1, GAPDH, ENO1, ALDOC, PDK1, LDHA, HK2, SHMT2, ENO2, HK3, SDS, PGLS, G6PD, and PRIA (Figure 3(c)). The expression levels 


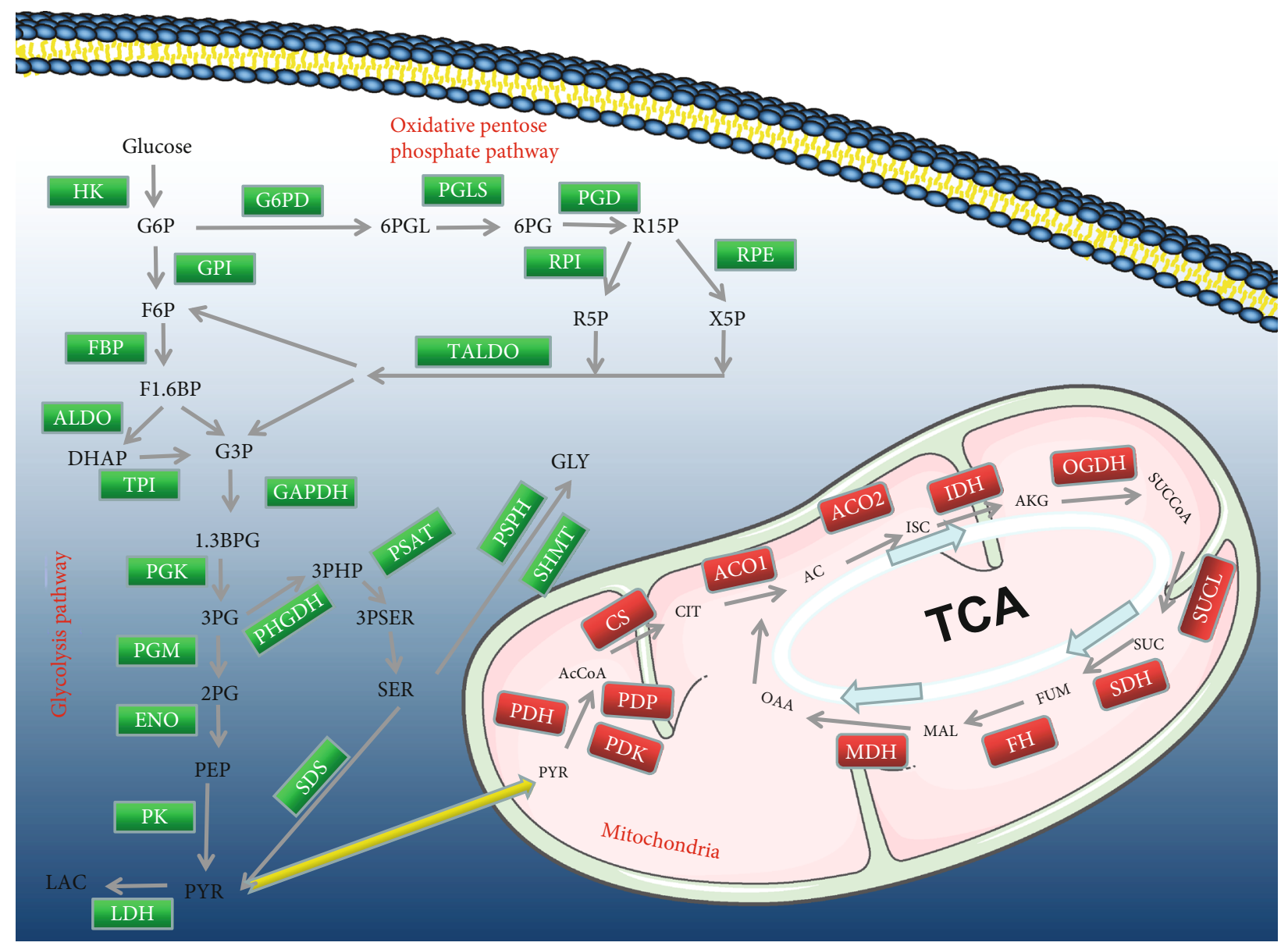

Figure 1: All genes that are involved in the glycometabolism.

of genes involved in glucose metabolism were consistent with this result (Figure 3(d)). Therefore, we speculated that these 43 differentially expressed glycolysis-related genes may be related to the occurrence of ccRCC.

3.2. Gene Coexpression Network Analysis and the Search for Potential Transcriptional Factors. Coexpression network revealed that glycolysis-related genes exhibited a strong coexpression relationship. We used the GEPIA website to analyze the correlation between GAPDH and TPI1 and found that they were very significant $(R=0.61)$. When the GAPDH gene was upregulated, TPI1 was also most likely upregulated (Figure 4(a)). As shown in Figure 4(b), transcription factors differentially expressed in the cancer and normal control groups were observed in the heat map. We analyzed all the differentially expressed transcriptional factors, and the volcano plot revealed that 41 transcription factors were upregulated and 19 transcription factors were downregulated in ccRCC $\left(p<0.05, \quad \log _{2}\right.$ fold - change $\left.>1\right)$ (Figure 4(c)). To investigate transcriptional factors upstream of the main differentially expressed genes in ccRCC, we generated a transcriptional factor network and observed that several core transcriptional factors were associated with the differentially expressed genes namely, FLI1,
ETS1, SREBF2, PML, CEBPB, RUNX1, MYBL2, CENPA, FOXM1, and LMNB1 (Figure 4(d)).

\subsection{Categories Determined by Consensus Clustering Were} Closely Correlated to the Clinical Outcomes and Clinicopathological Features. ConsensusClusterPlus was used to group 539 cancer tissues. Based on the similarity in the expression of glycolysis-related genes, $k=2$ had the smallest CDF value; therefore, the cancer tissues were divided into two groups (Figures 5(a) and 5(b)). We analyzed the two subgroups to validate our classification by PCA, and the results showed that both cluster 1 and cluster 2 could also be clustered independently (Figure 5(c)). Furthermore, we analyzed the clustering results and OS curves of 539 ccRCC patients and found that the cluster 1 subgroup was associated with a significantly shorter OS than the cluster 2 subgroup (Figure 5(d)). Moreover, we found that most of the glycolysis-related genes were highly expressed in the cluster 1 subgroup. Compared to the cluster 2 subgroup, the cluster 1 subgroup was significantly correlated with a higher stage, higher grade, higher $\mathrm{T}$ status, higher $\mathrm{M}$ status, and fustat (Figure 5(e)).

3.4. Drug Sensitivity and Pathway Analysis. Drug sensitivity was analyzed using Genomics of Drug Sensitivity in Cancer 


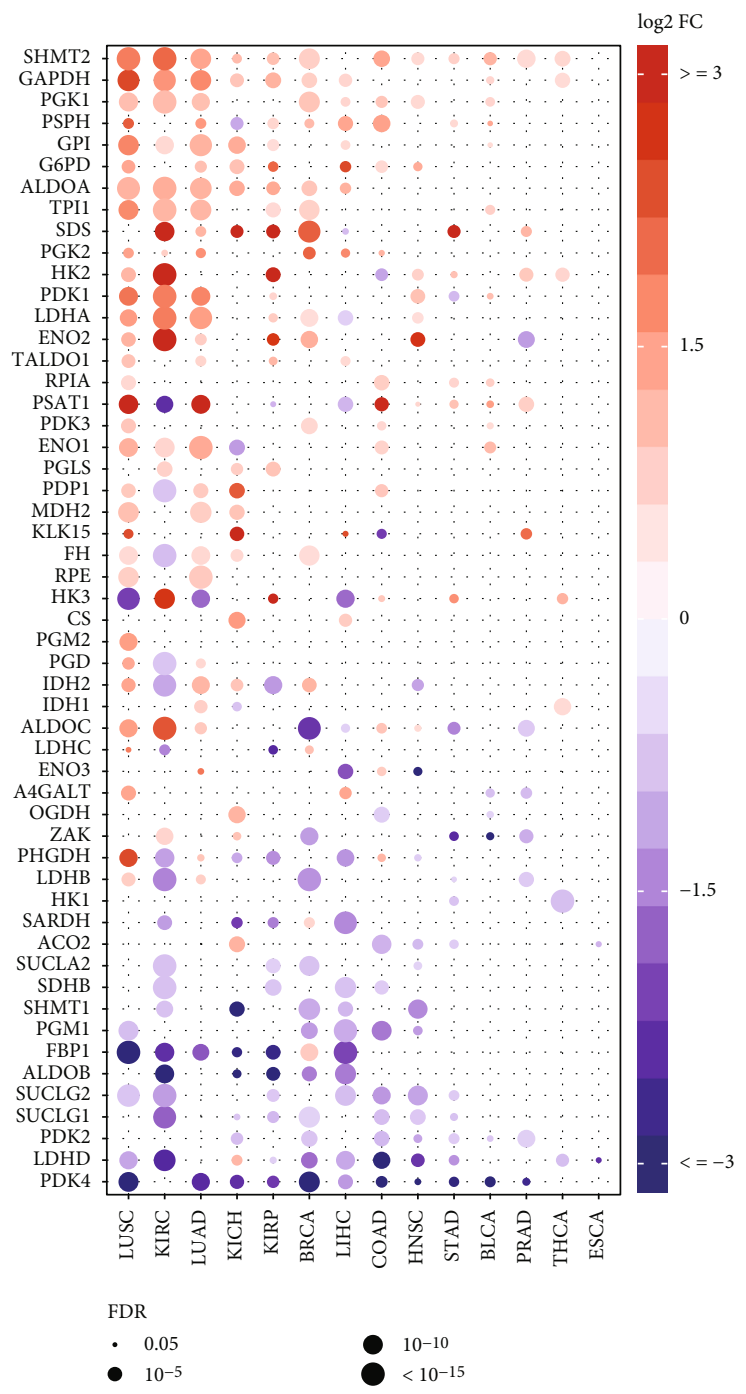

(a)

FIgUre 2: Continued. 


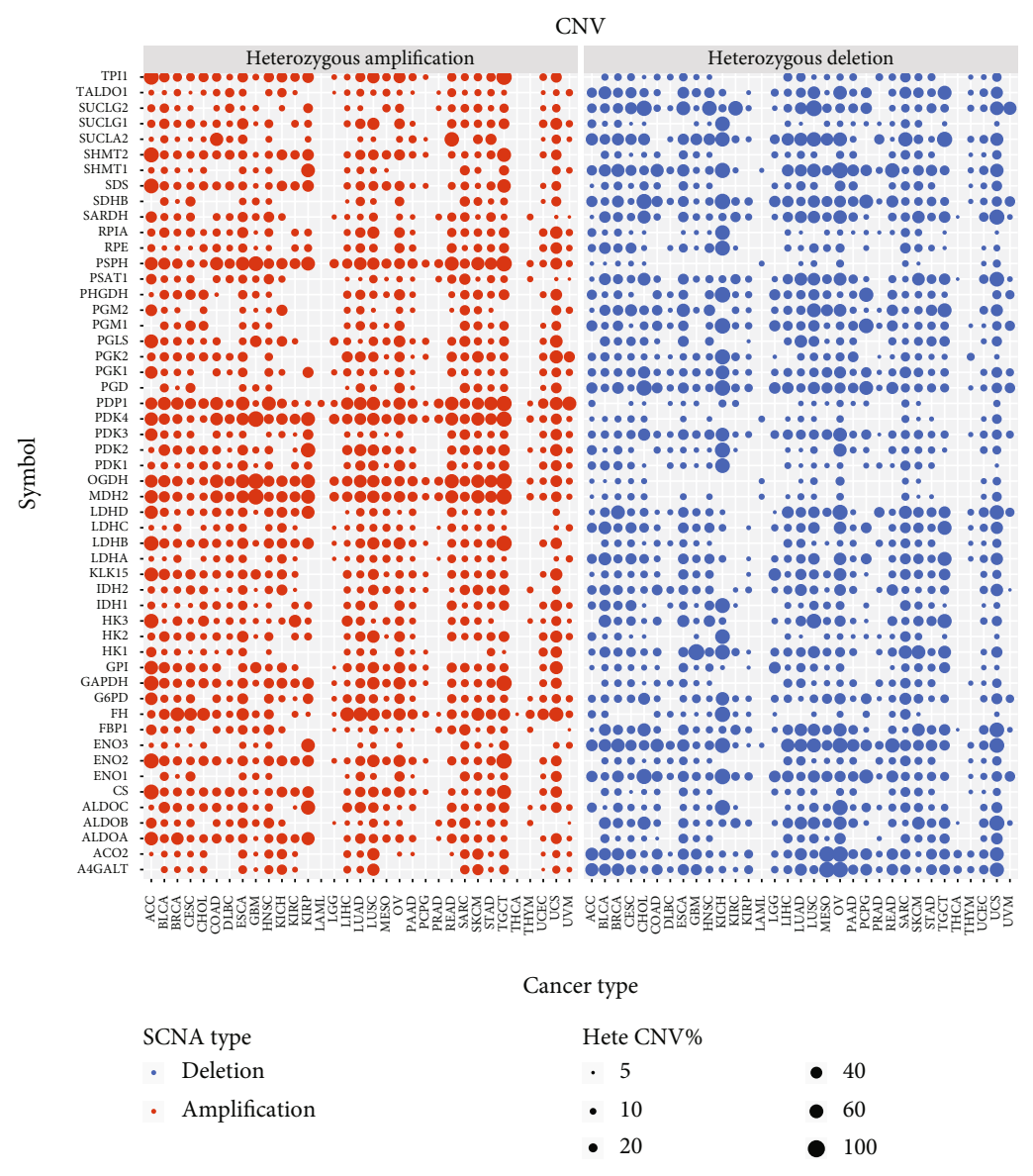

(b)

Figure 2: Continued. 
SNV
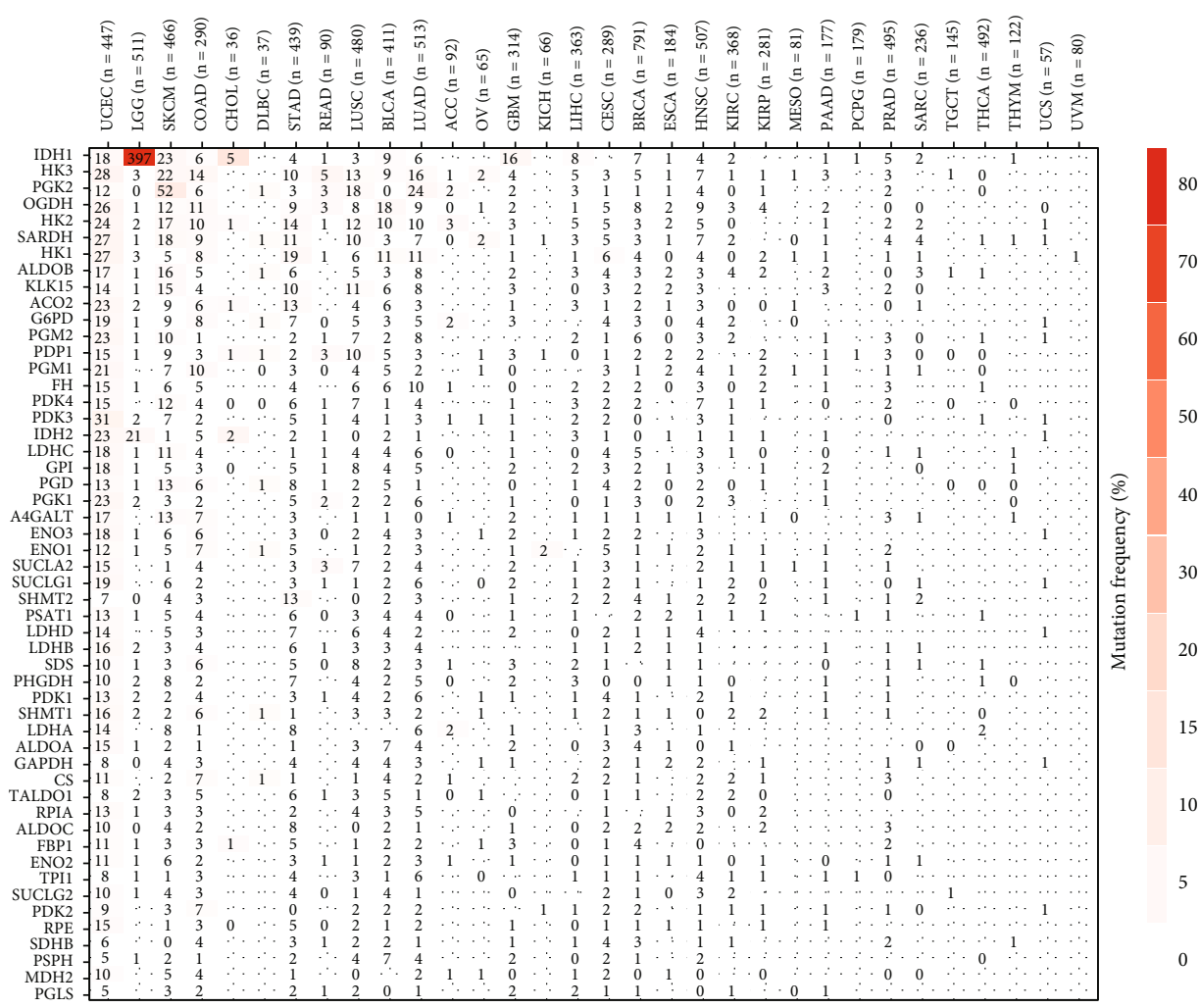

(c)

Figure 2: Alterations in the expression genes involved in glycolysis. (a) The gene expression alterations in 14 cancer types. (b) The CNV alteration frequency of genes across cancer types. The left part of each grid shows the amplification frequency, and the right part shows the deletion frequency. (c) The SNV alteration frequency of genes across cancer types.

(GDSC; https://www.cancerrxgene.org/) [25]. The expression of some differentially expressed genes, such as PRIA, SUCLG2, ENO2, and G6PD, was positively correlated with drug sensitivity. The high expression of G6PD was positively correlated with sensitivity to 5-fluorouracil, CX-5461, AT7519, and PHA-793887, and the high expression of HK3 was negatively correlated with sensitivity to 5-fluorouracil, ATRA, and I-BET-762 (Figure 6(a)). Pathway analysis revealed that PSAT1 and SDHB could activate the cell cycle, RPIA could activate the DNA damage response, and HK3 could activate apoptosis and EMT, while PDK2 could inhibit apoptosis (Figure 6(c)).

3.5. Establishment of a Risk Signature and Its Prognostic Value in ccRCC. To explore the prognostic role of differentially expressed glycolysis-related genes in ccRCC, we subjected gene expression data from TCGA to univariate Cox regression analysis. Twenty-five genes were found to have prognostic value with respect to ccRCC $(p<0.05)$ (Figure 6(b)). Among these, IDH1 $(\mathrm{HR}=1.022,95 \% \mathrm{CI}=$ $1.008-1.037)$, G6PD $(\mathrm{HR}=1.043,95 \% \mathrm{CI}=1.028-1.057)$, $\mathrm{HK} 3 \quad(\mathrm{HR}=1.151, \quad 95 \% \mathrm{CI}=1.089-1.217), \quad \mathrm{ENO} 2$ $(\mathrm{HR}=1.088,95 \% \mathrm{CI}=1.004-1.012), \mathrm{ENO} 3 \quad(\mathrm{HR}=1.030$, $95 \% \mathrm{CI}=1.007-1.054), \mathrm{RPIA}(\mathrm{HR}=1.103,95 \% \mathrm{CI}=1.041$ $-1.170)$, PSAT1 $\quad(\mathrm{HR}=1.016, \quad 95 \% \mathrm{CI}=1.010-1.023)$,
GAPDH $\quad(\mathrm{HR}=1.000, \quad 95 \% \mathrm{CI}=1.000-1.001), \quad$ TALDO1 $(\mathrm{HR}=1.010, \quad 95 \% \mathrm{CI}=1.004-1.016), \quad$ and $\quad$ PGLS $(\mathrm{HR}=1.031,95 \% \mathrm{CI}=1.017-1.046)$ - upon being expressed at high levels - resulted in a short survival duration in ccRCC patients. On the contrary, SHMT1 $(\mathrm{HR}=0.971,95 \% \mathrm{CI}=$ $0.956-0.987)$, ENO1 ( $\mathrm{HR}=0.999,95 \% \mathrm{CI}=0.999-1.000)$, ACO1 $\quad(\mathrm{HR}=0.955, \quad 95 \% \mathrm{CI}=0.921-0.991), \quad \mathrm{PDK} 4$ $(\mathrm{HR}=0.997,95 \% \mathrm{CI}=0.996-0.999)$, SUCLG2 $(\mathrm{HR}=0.961$ , $95 \% \mathrm{CI}=0.941-0.982), \quad \mathrm{SDHB} \quad(\mathrm{HR}=0.958,95 \% \mathrm{CI}=$ $0.937-0.978), \mathrm{OGDH}(\mathrm{HR}=0.981,95 \% \mathrm{CI}=0.972-0.991$ ) , PDK2 $(\mathrm{HR}=0.915,95 \% \mathrm{CI}=0.880-0.950)$, SUCLA2 $(\mathrm{HR}=0.917,95 \% \mathrm{CI}=0.887-0.947), \mathrm{ACO} 2 \quad(\mathrm{HR}=0.979$, $95 \% \mathrm{CI}=0.971-0.988), \mathrm{FBP} 1 \quad(\mathrm{HR}=0.982,95 \% \mathrm{CI}=0.973$ -0.991), PGK1 $\quad(\mathrm{HR}=0.996, \quad 95 \% \mathrm{CI}=0.994-0.998)$, PGM2 $\quad(\mathrm{HR}=0.894, \quad 95 \% \mathrm{CI}=0.845-0.945), \quad$ PKLR $(\mathrm{HR}=0.978, \quad 95 \% \mathrm{CI}=0.959-0.998), \quad$ and $\quad$ LDHD $(\mathrm{HR}=0.955,95 \% \mathrm{CI}=0.922-0.989)$ - upon being expressed at high levels-resulted in a longer survival duration in patients with ccRCC.

Subsequently, we selected 20 genes that were identified as being significant in the univariate Cox analysis $(p<0.01)$ for the LASSO regression analysis. Thirteen genes were selected to generate the risk signature, and the coefficients obtained from the LASSO algorithm were used to calculate the risk score (Figures 6(d) and 6(e)). We then stratified the ccRCC 


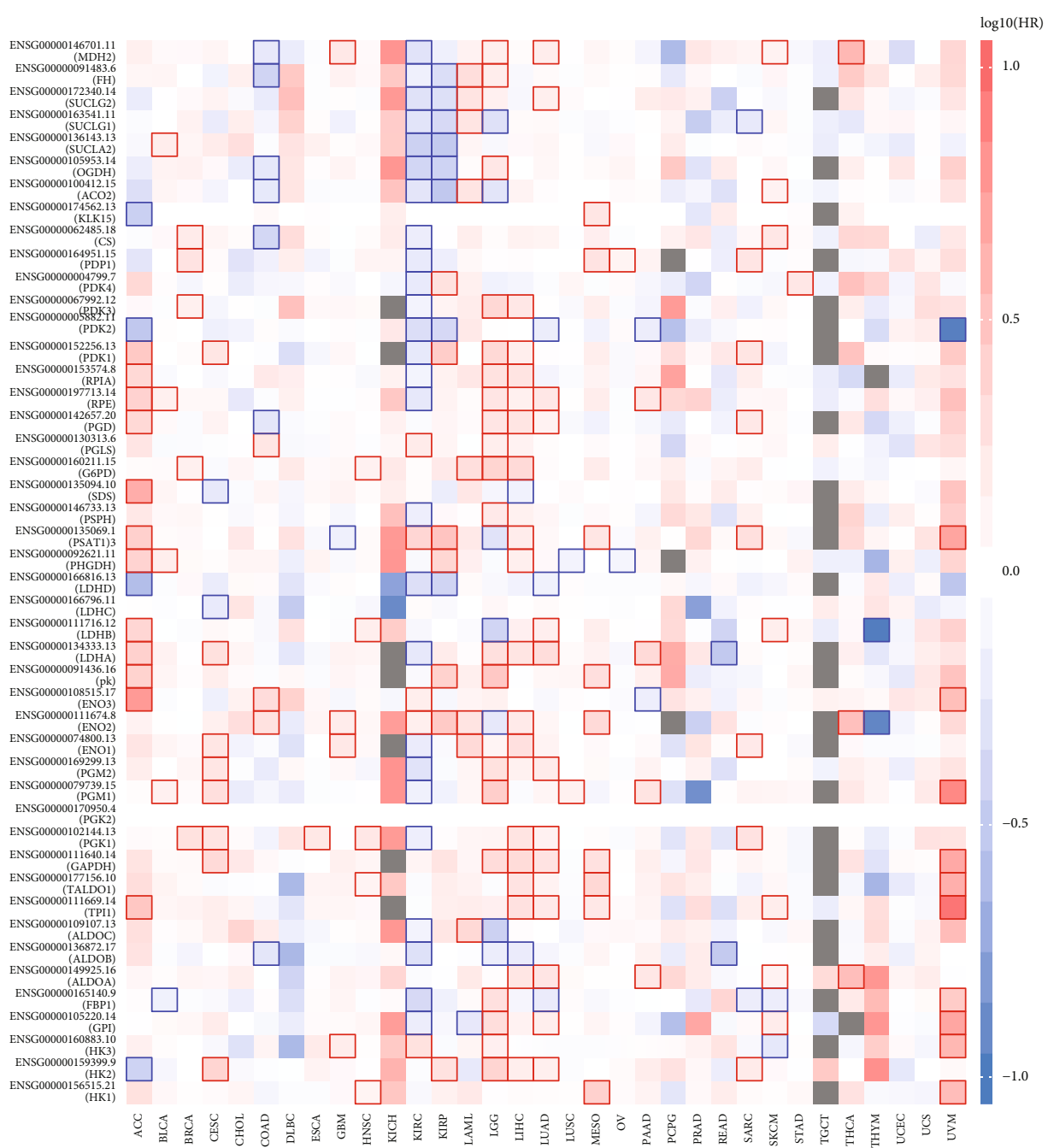

(a)

FIgURe 3: Continued. 


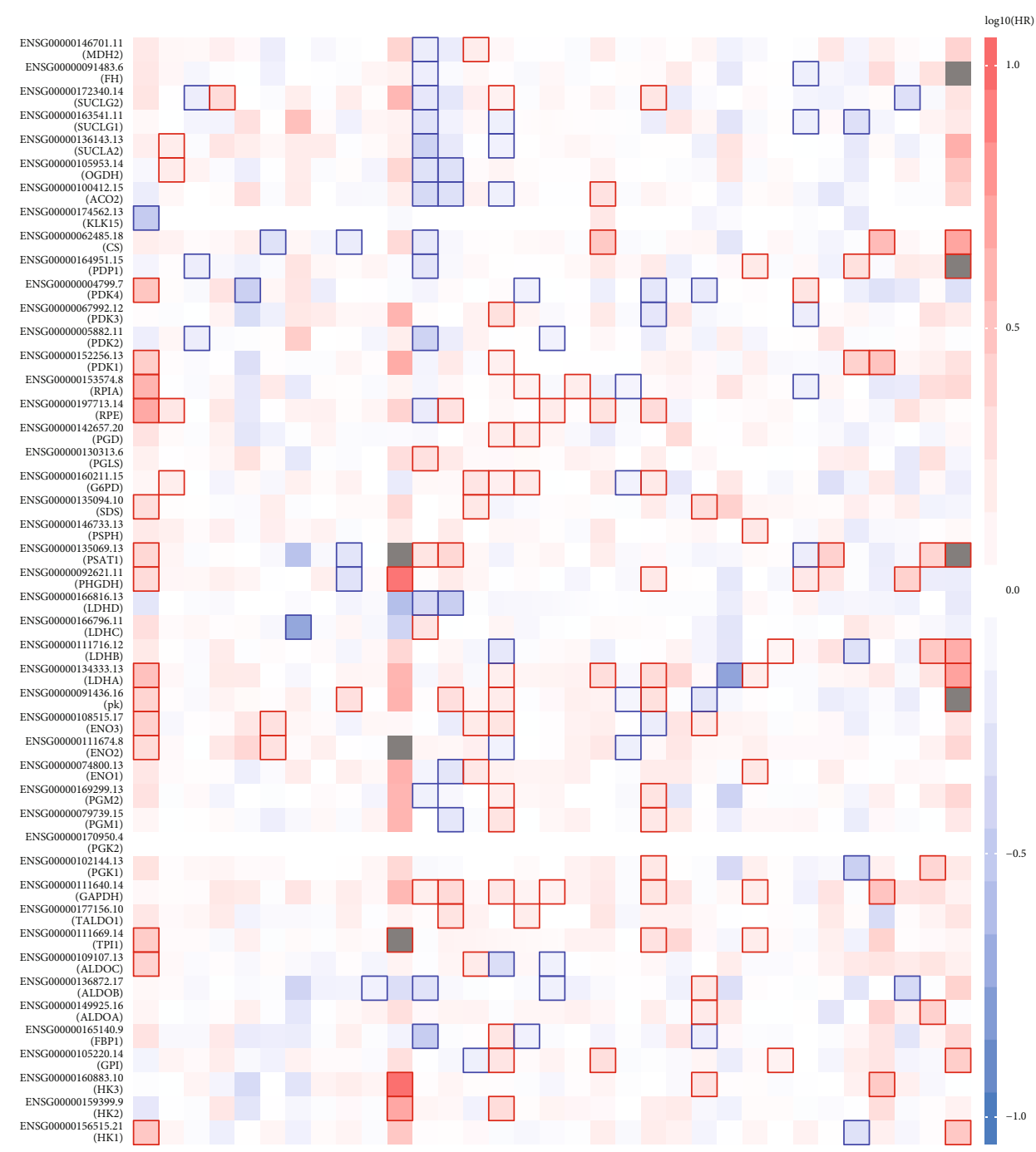

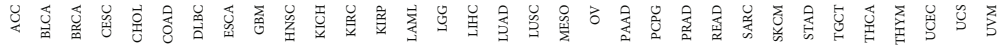

(b)

Figure 3: Continued. 


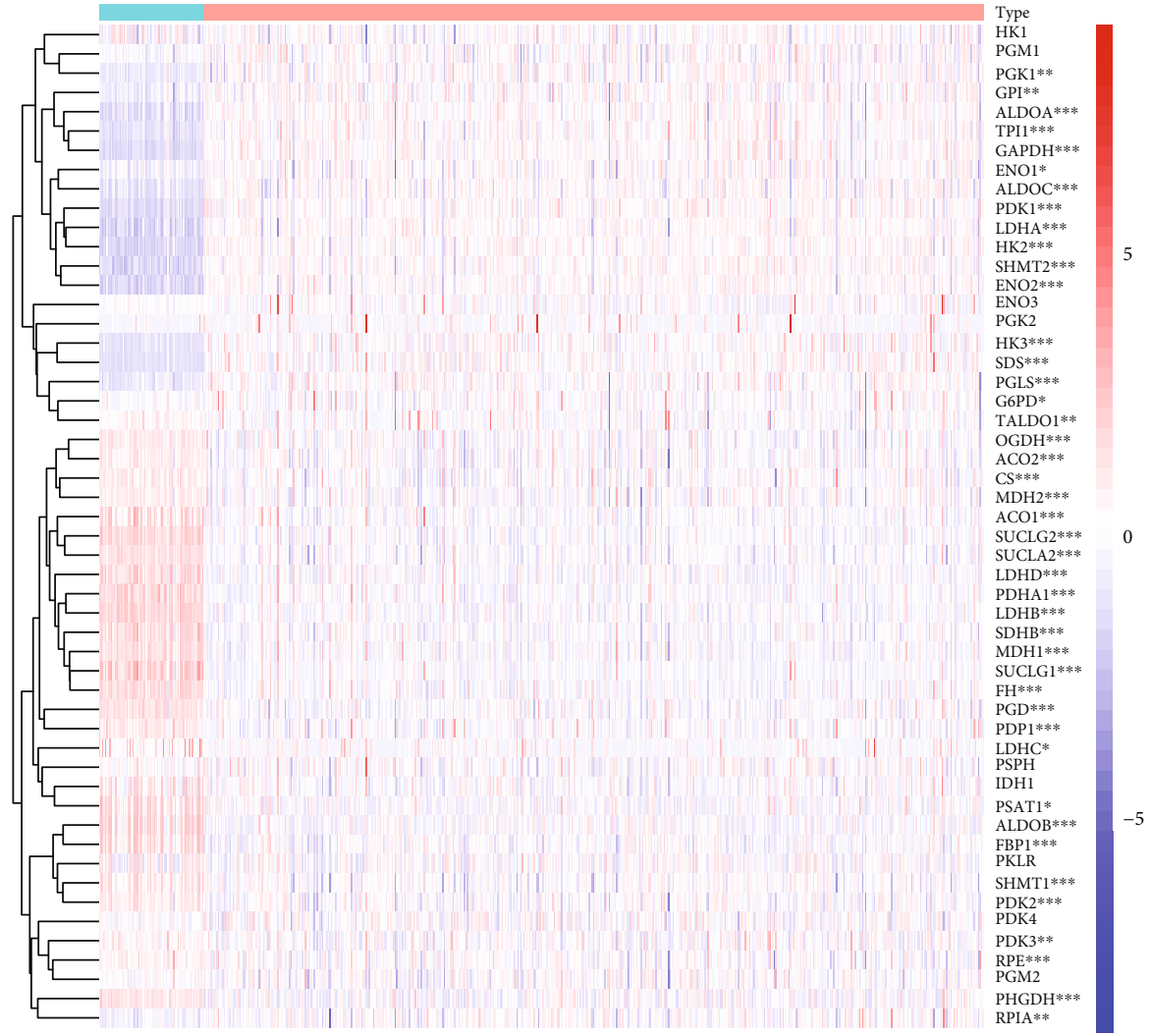

Type

(c)

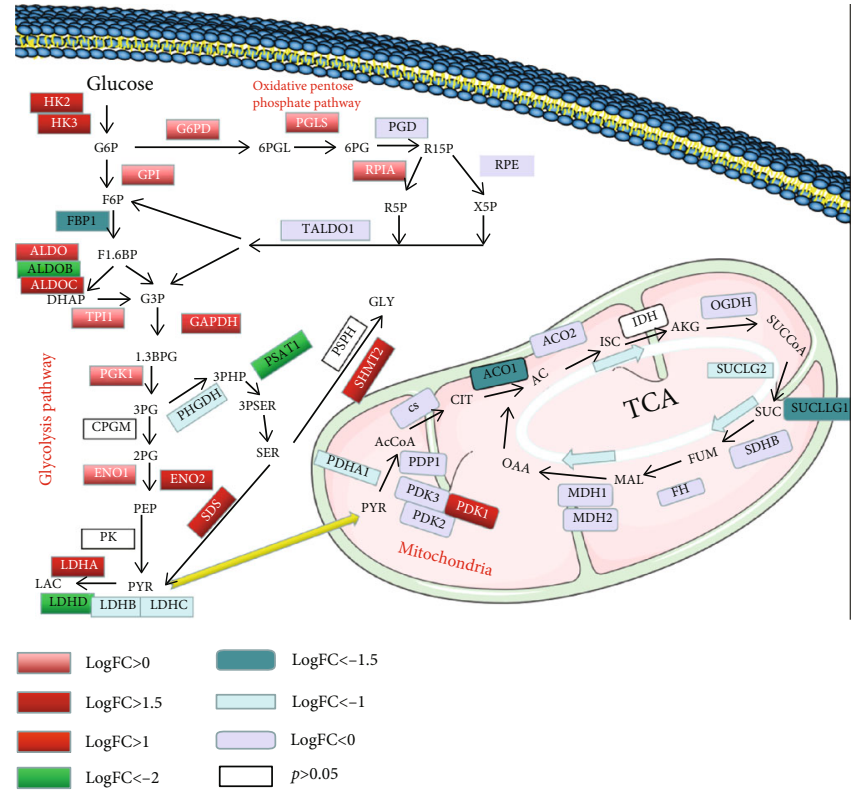

(d)

FIGURE 3: Relationship between gene expression levels and hazard ratio (HR) of ccRCC. (a) The relationship between gene expression levels and overall survival (OS). (b) The relationship between gene expression levels and disease free survival (DFS). (c) The expression levels of 52 genes in 539 ccRCC samples and 72 normal control samples. (d) The expression levels of all genes involved in glycometabolism in ccRCC. ${ }^{*} p<0.05,{ }^{* *} p<0.01$, and ${ }^{* * *} p<0.001$. 


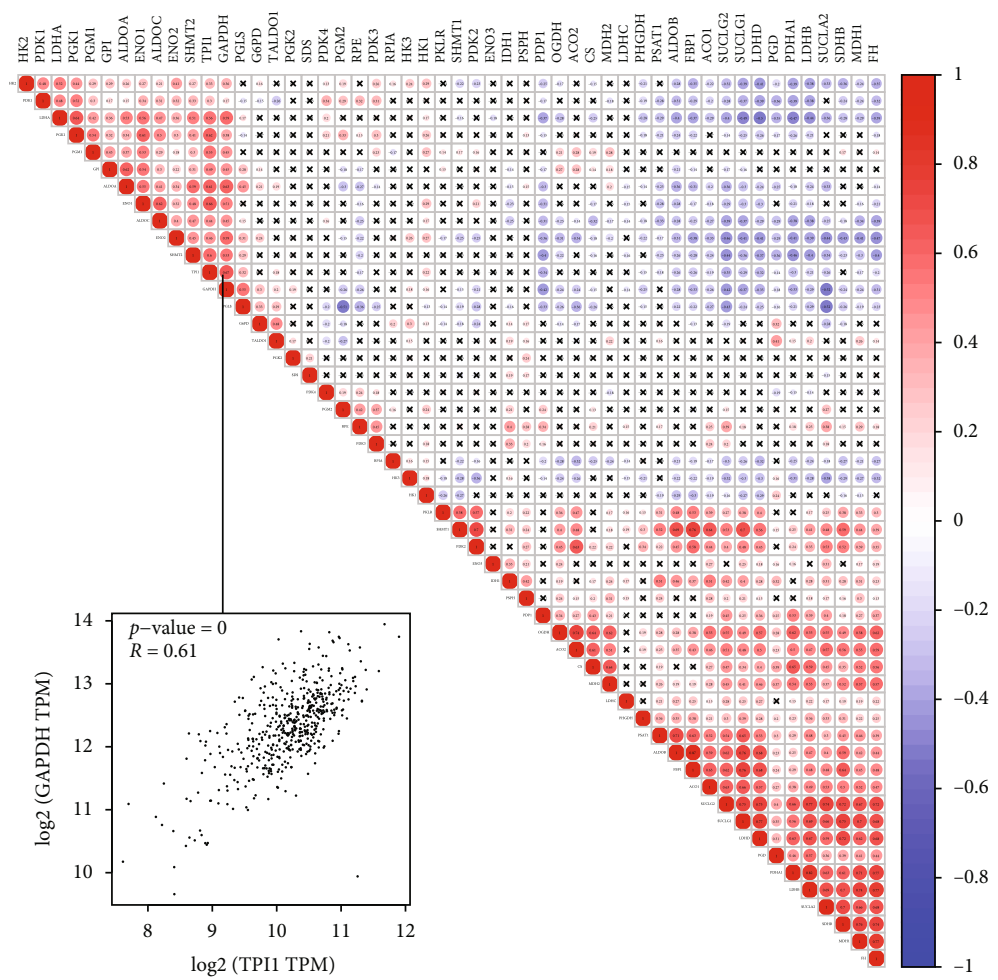

(a)

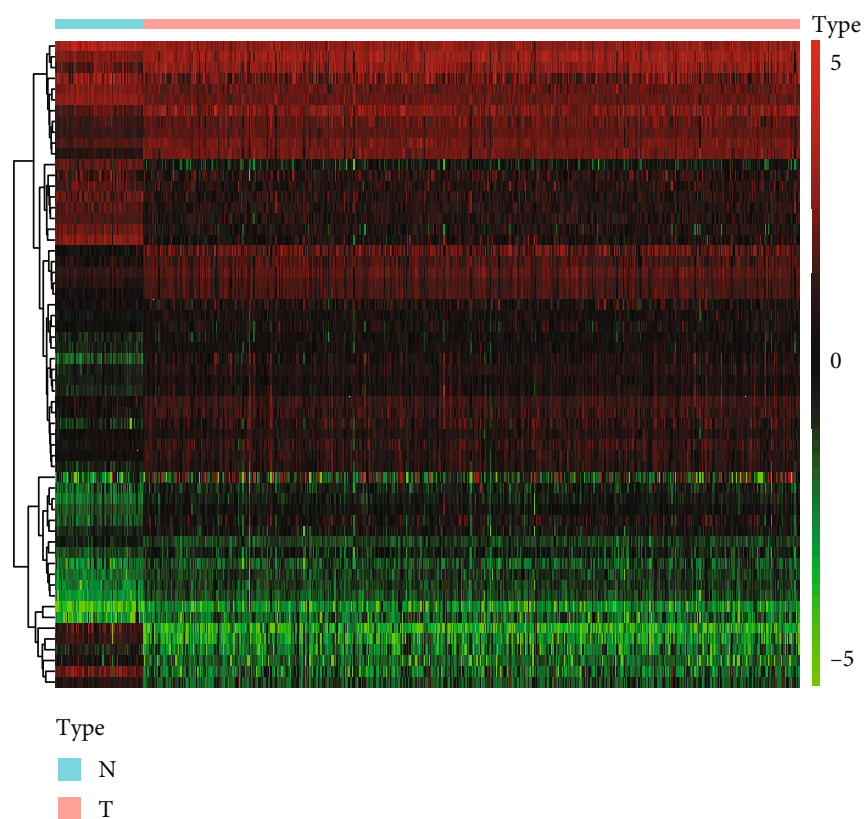

(b)

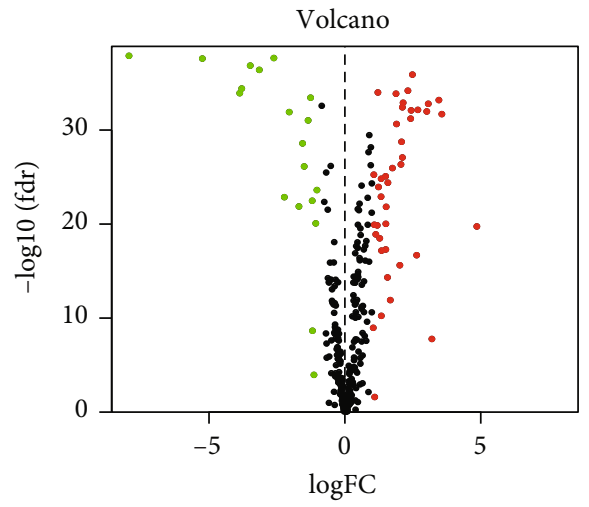

(c)

Figure 4: Continued. 


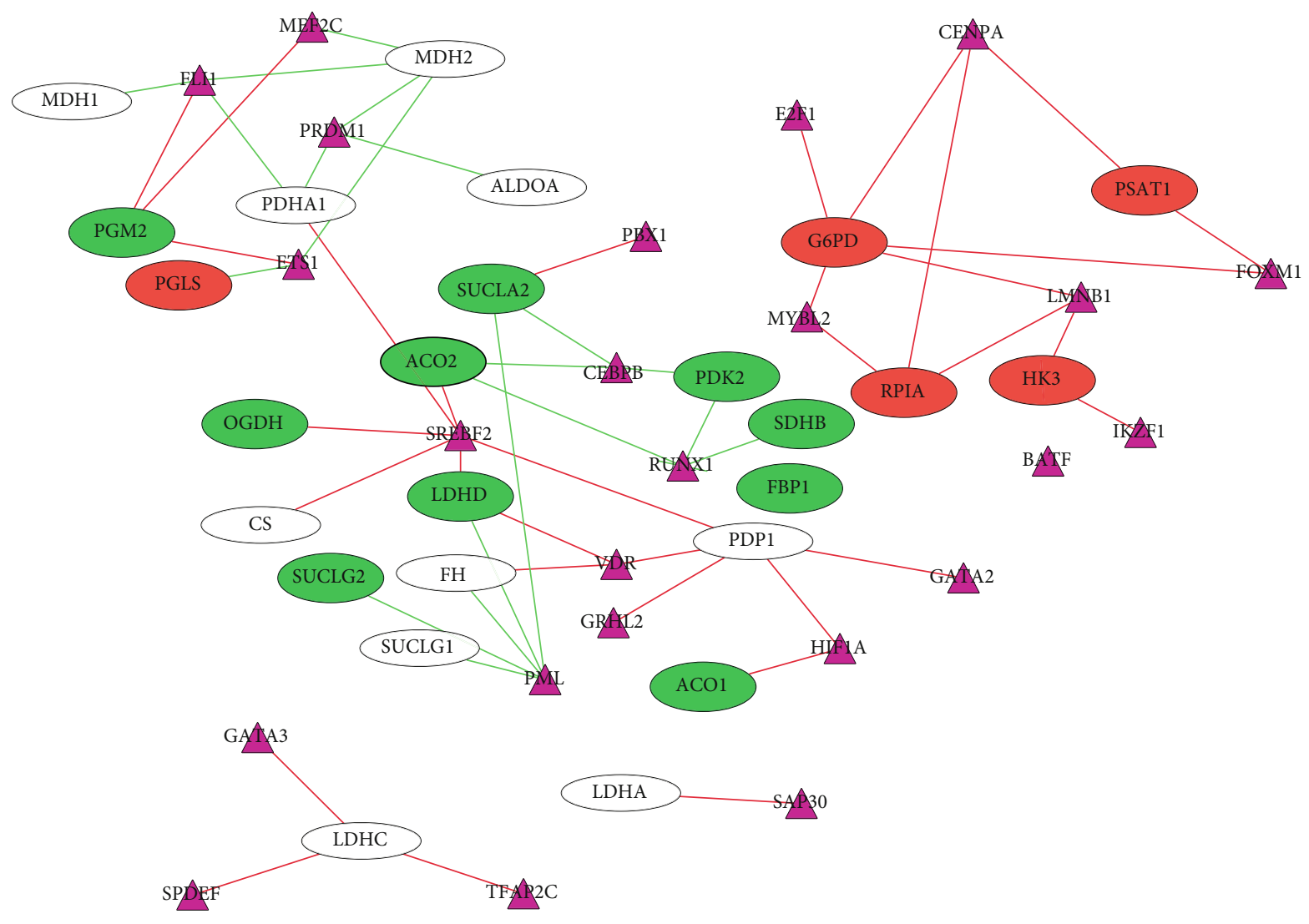

(d)

Figure 4: Coexpression network for the glycolysis-related genes and the transcriptional factor network between ccRCC group and normal control group. (a) Coexpression network for glycolysis-related genes and the correlation between GAPDH and TPI1. (b) The expression levels of transcriptional factors in 539 ccRCC samples and 72 normal control samples. (c) Volcano plot of all transcriptional factors; red color represents transcriptional factors with high expression, green color represents transcriptional factors with low expression, and black color represents transcription factors with no differential expression. (d) The transcriptional factor upstream of the main differentially expressed glycolysis-related genes in ccRCC. The purple triangle represents the transcriptional factors, the red color represents upregulated glycolysis-related genes, and the green color represents downregulated glycolysis-related genes.

patients into the low-risk and high-risk groups according to the median risk score; the results indicated that patients in the high-risk group had a lower survival rate (Figure $7(\mathrm{a})$ ). ROC curve analysis was performed to predict the risk scores and survival rate of ccRCC patients; the area under the curve (AUC) was found to be 0.731 . The results showed that the risk scores could predict the survival rate of patients with ccRCC (Figure 7(b)).

3.6. The Risk Signature Was Closely Related to the Clinicopathological Characteristics of ccRCC. We studied the relationship between the 13 selected genes in the high- and low-risk groups - from TCGA — and the pathological characteristics of cancer, including age, stage, grade, $T$ status, $M$ status, and $\mathrm{N}$ status. We found that these 13 genes were closely related to the pathological characteristics of cancer. Moreover, compared with patients in the low-risk group, ccRCC patients in the high-risk group exhibited higher expression of RPIA, G6PD, PSAT1, ENO2, HK3, and IDH1 and lower expression of PDK4, PGM2, PGK1, FBP1, OGDH, SUCLA2, and SUCLG2 (Figures $7(\mathrm{c})$ and $7(\mathrm{~h})$ ).
We performed univariate and multivariate Cox regression analyses on TCGA data. Univariate Cox regression analyses indicated that the risk score, age, stage, grade, $\mathrm{T}$ status, and $\mathrm{M}$ status were all related to OS. As the risk score, age, stage, grade, $\mathrm{T}$ status, and $\mathrm{M}$ status increased, the risk increased. Multivariate Cox regression analyses indicated that the risk score, age, stage, and grade were independent risk factors (Figures $7(\mathrm{~d})$ and $7(\mathrm{e})$ ). Furthermore, as the risk scores increased, the risk of developing cancer and the number of deaths increased (Figures $7(\mathrm{f})$ and $7(\mathrm{~g})$ ). Based on these results, we conclude that the established risk signature is closely correlated with the clinicopathological characteristics of ccRCC.

\section{Discussion}

Glycolysis is a natural process that occurs in normal tissues under hypoxic conditions. Glycolysis has also been observed in areas of malignant tumors with sufficient oxygen. To enable the proliferation of tumor cells and to enable the formation of a membrane structure, it is necessary to produce a 

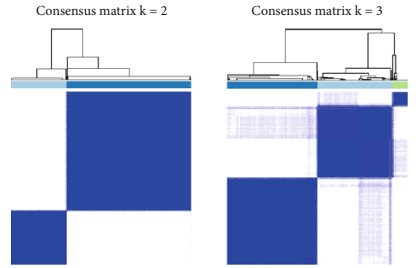

- 1

ㅁ 1

- 2

- 2

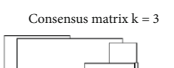

Consensus matrix $k=5$
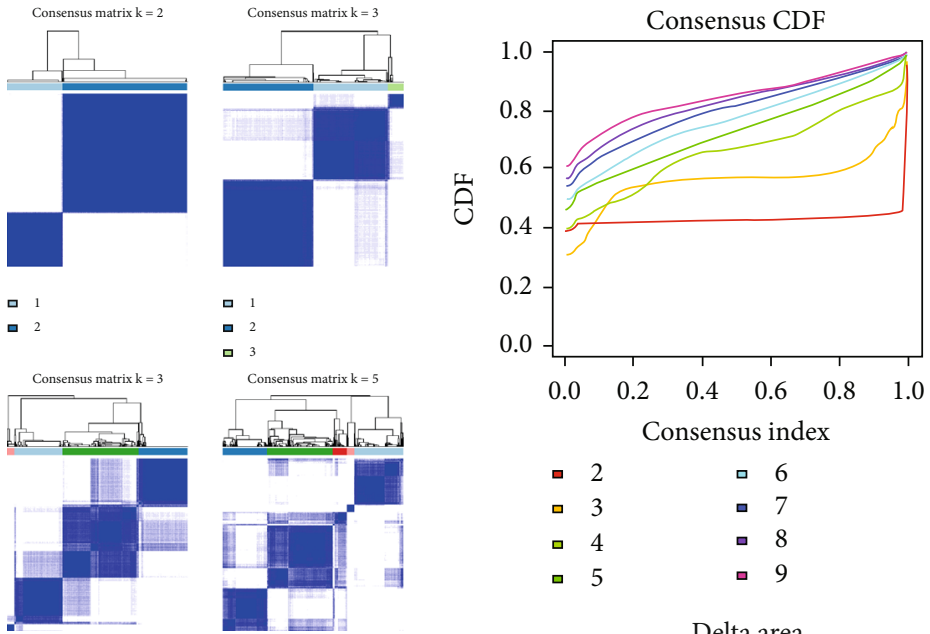

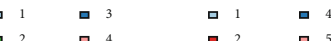

$$
4
$$

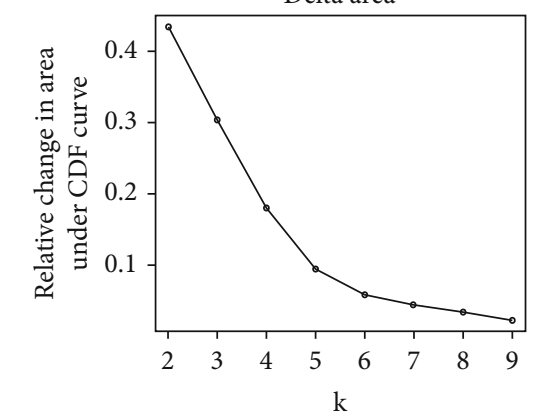

(a)

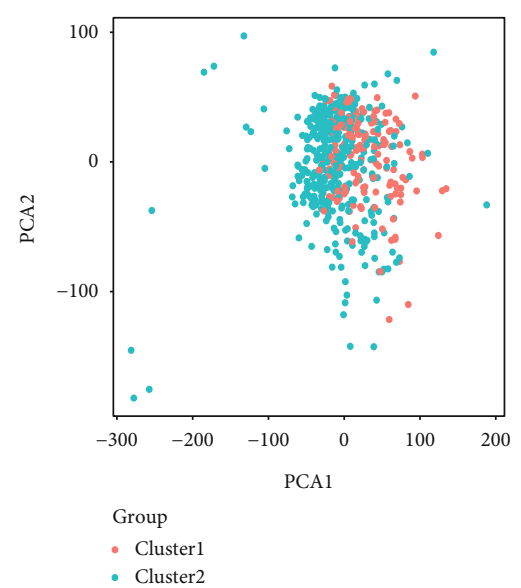

(b)

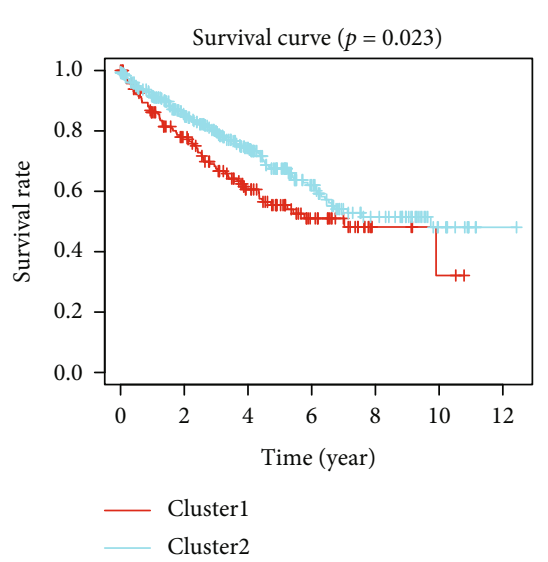

(c)

(d)

Figure 5: Continued. 


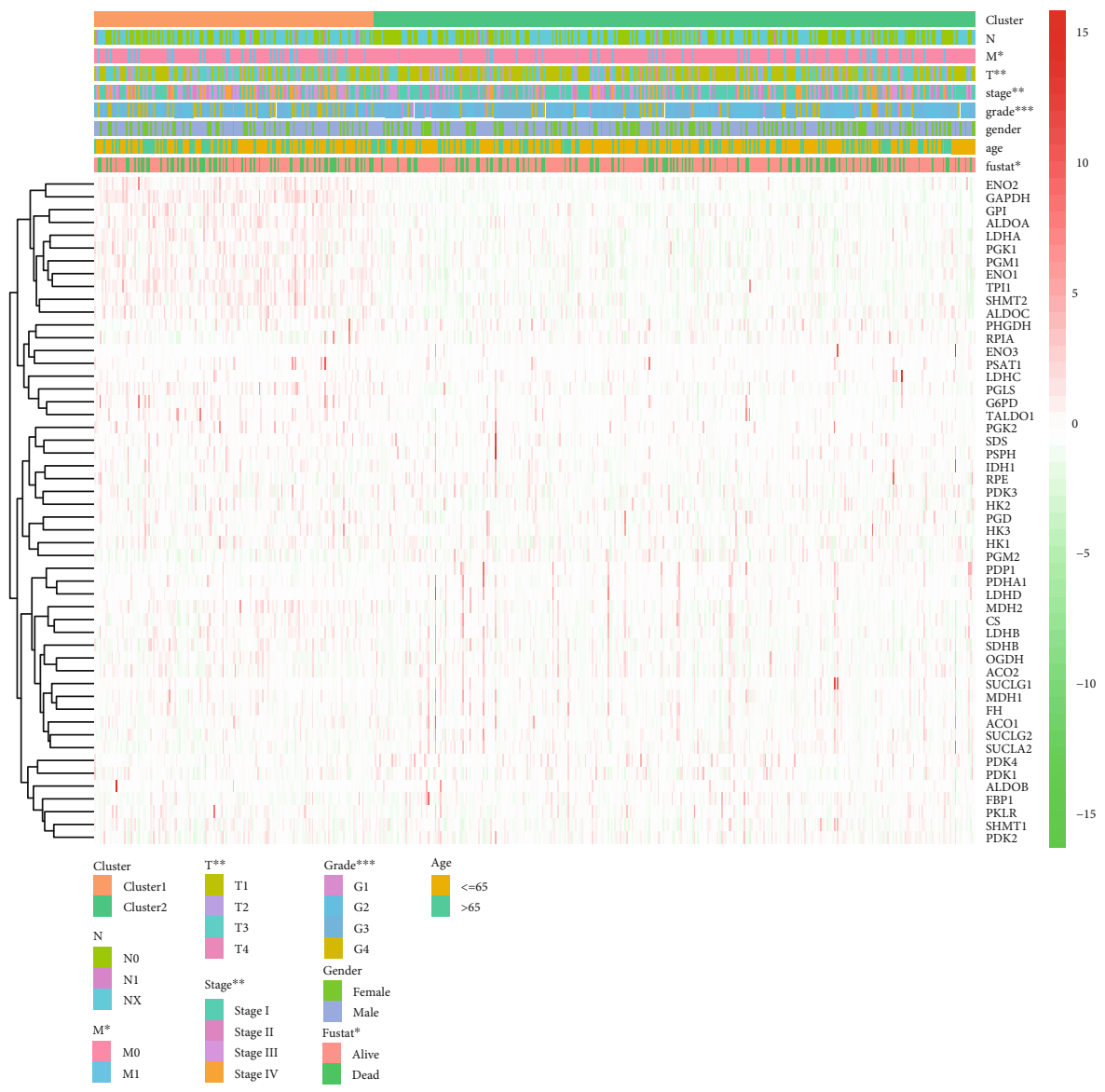

(e)

FIgURE 5: Identification of consensus clusters based on glycolysis-related genes and overall survival of ccRCC in the cluster $1 / 2$ subgroups. (a) Consensus clustering matrix for $K=2-5$. (b) Consensus clustering cumulative distribution function (CDF) for $K=2-9$, relative change in area under the CDF curve for $K=2-9$. (c) PCA of the total genes in TCGA dataset. (d) Kaplan-Meier overall survival (OS) curve for 539 ccRCC patients. (e) Based on the results of this cluster analysis, heat map shows the correlation with clinicopathological characteristics.

large number of fatty acids, a process that requires aerobic glycolysis, also known as the Warburg effect. This phenomenon is very common in malignant tumors [26-29]. Glycolysis is very important for tumors, and tumor cells exhibit high expression of key enzymes involved in the process of glycolysis to generate more energy. In addition, tumor cells can also produce energy through the fermentation of lactic acid, a product of glycolysis [30]. The Warburg effect and glycolytic genes have been studied in lung cancer, breast cancer, ovarian cancer, gastric cancer, liver cancer, colorectal cancer, esophageal cancer, prostate cancer, bladder cancer, and other tumors [11-19].

A recent study has shown that the Warburg effect in ccRCC is more pronounced than that in other tumors [31]. A report based on metabolic atlas showed that ccRCC is characterized by an increase in the level of metabolites during glycolysis and a decrease in the level of metabolites during oxidative phosphorylation, suggesting that glycolysis is active in ccRCC [21]. Therefore, we chose to study the expression of glycolysis-related genes in ccRCC patients to identify biomarkers that can predict the prognosis of the disease. Unlike many previous studies that may be limited to a single gene $[32,33]$, our research attempts to further understand the occurrence and development of tumors by studying the entire biological pathway. This new type of research is currently being respected and has made progress [34-36].

Our study included 539 ccRCC tissue samples and 72 normal control tissue samples from the TCGA dataset. We analyzed 53 glycolysis-related genes and identified 43 differentially expressed genes between the ccRCC and normal control groups. In addition, we identified 25 genes related to the prognosis of ccRCC patients using univariate Cox analysis. Finally, we used LASSO regression analysis to construct a risk model consisting of 13 genes: RPIA, G6PD, PSAT1, ENO2, HK3, IDH1, PDK4, PGM2, PGK1, FBP1, OGDH, SUCLA2, and SUCLG2.

ENO2 is a key gene in glycolysis, which catalyzes the dehydration of 2-phosphoglyceric acid to produce phosphoenolpyruvate. It can promote cell growth, upregulate glycolysis-related genes, and activate Akt signaling pathway through phosphorylation of glycogen synthase kinase $3 \beta$, so as to induce cell proliferation and glycolysis [37]. Increased expression of ENO2 has been found in many types of tumors. The high expression of ENO2 in glioma and colorectal cancer is related to glycolysis in the tumor cells [38, 39]. The 


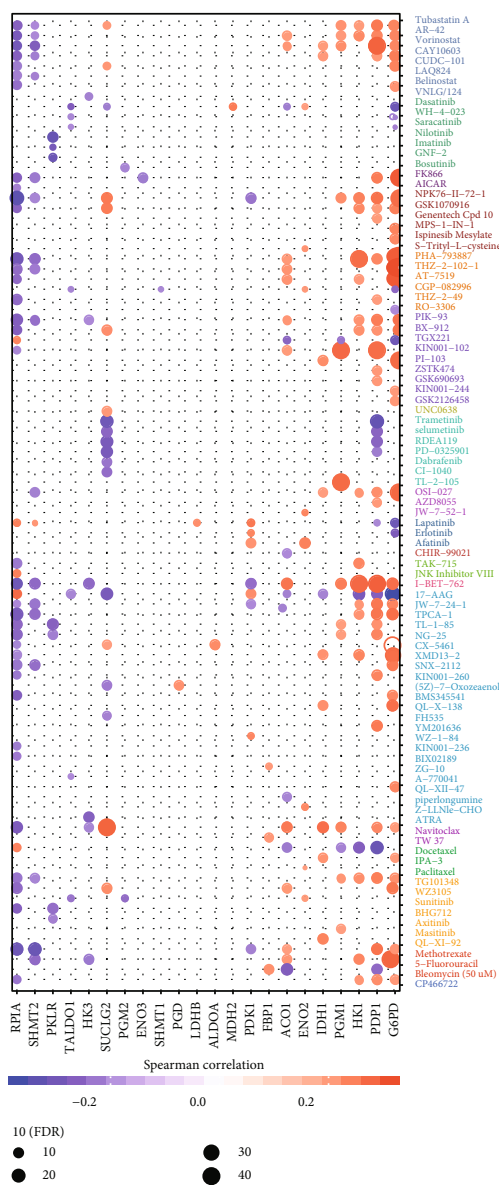

\begin{tabular}{lcc} 
& pvalue & Hazard ratio \\
SHMT1 & $<0.001$ & $0.971(0.956-0.987)$ \\
SHMT2 & 0.190 & $1.005(0.998-1.012)$ \\
IDH1 & 0.002 & $1.022(1.008-1.037)$ \\
PGD & 0.105 & $1.014(0.997-1.031)$ \\
ENO1 & 0.042 & $0.999(0.999-1.000)$ \\
G6PD & $<0.001$ & $1.043(1.028-1.057)$ \\
PHGDH & 0.829 & $0.998(0.976-1.020)$ \\
ACO1 & 0.014 & $0.955(0.921-0.991)$ \\
HK3 & $<0.001$ & $1.151(1.089-1.217)$ \\
CS & 0.454 & $0.993(0.976-1.011)$ \\
PDK4 & $<0.001$ & $0.997(0.996-0.999)$ \\
PDK1 & 0.090 & $0.945(0.885-1.009)$ \\
MDH1 & 0.263 & $0.992(0.977-1.006)$ \\
ENO2 & $<0.001$ & $1.008(1.004-1.012)$ \\
SUCLG1 & 0.306 & $0.991(0.974-1.008)$ \\
SUCLG2 & $<0.001$ & $0.961(0.941-0.982)$ \\
GP1 & 0.496 & $0.998(0.992-1.004)$ \\
SDHB & $<0.001$ & $0.958(0.937-0.978)$ \\
ALDOA & 0.786 & $1.000(0.999-1.001)$ \\
OGDH & $<0.001$ & $0.981(0.972-0.991)$ \\
ENO3 & 0.012 & $1.030(1.007-1.054)$ \\
PDK2 & $<0.001$ & $0.915(0.880-0.950)$ \\
RPIA & $<0.001$ & $1.103(1.041-1.170)$ \\
PGM1 & 0.275 & $0.993(0.981-1.006)$ \\
SUCLA2 & $<0.001$ & $0.917(0.887-0.947)$ \\
ALDOB & 0.184 & $1.000(1.000-1.001)$ \\
SDS & 0.557 & $0.993(0.971-1.016)$ \\
TPI1 & 0.630 & $1.000(0.999-1.001)$ \\
PDHA1 & 0.265 & $0.988(0.967-1.009)$ \\
LDHA & 0.373 & $0.999(0.998-1.001)$ \\
HK1 & 0.129 & $1.015(0.996-1.035)$ \\
ACO2 & $<0.001$ & $0.979(0.971-0.988)$ \\
LDHB & 0.704 & $1.000(0.997-1.002)$ \\
FBP1 & $<0.001$ & $0.982(0.973-0.991)$ \\
TALDHO1 & $<0.001$ & $1.010(1.004-1.016)$ \\
PDHC & 0.917 & $1.016(0.757-1.362)$ \\
PK2 & 0.254 & $1.008(0.995-1.021)$ \\
PGLS & $<0.001$ & $1.031(1.017-1.046)$ \\
PSAT1 & 0.189 & $0.963(0.911-1.019)$ \\
PSH & $<0.001$ & $1.016(1.010-1.023)$ \\
RPE & 0.344 & $0.971(0.915-1.031)$ \\
PGK1 & $<0.001$ & $0.996(0.994-0.998)$ \\
PGM2 & $<0.001$ & $0.894(0.845-0.945)$ \\
ALDOC & 0.066 & $0.993(0.986-1.000)$ \\
PKLR & 0.031 & $0.978(0.959-0.998)$ \\
LDHD & 0.010 & $0.955(0.922-0.989)$ \\
MAPDH & $<0.001$ & $1.000(1.000-1.001)$ \\
\hline 0.161 & $0.993(0.984-1.003)$ \\
\hline & $0.0985(0.968-1.002)$ \\
\hline
\end{tabular}

(a)

(b)

Figure 6: Continued. 


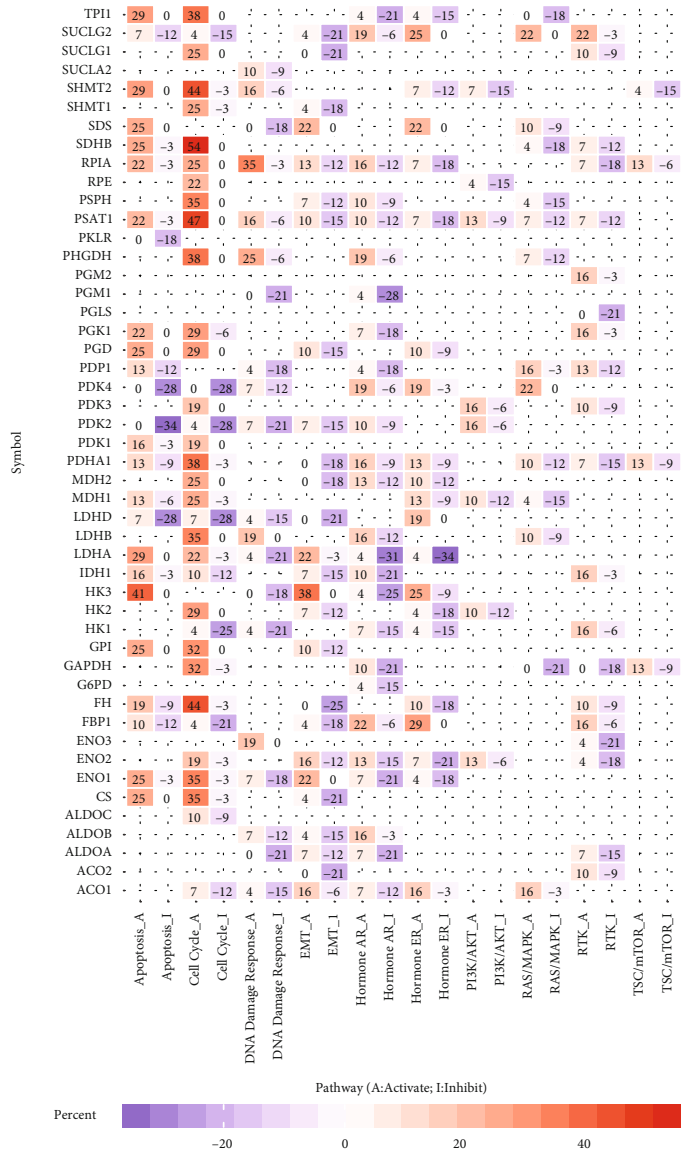

(c)

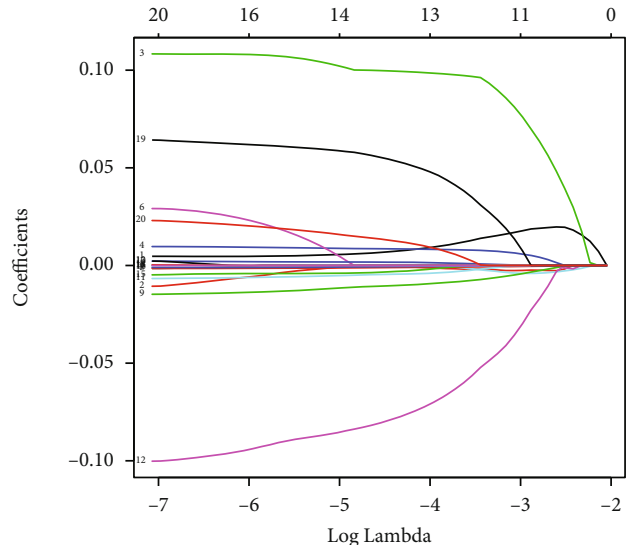

(d)

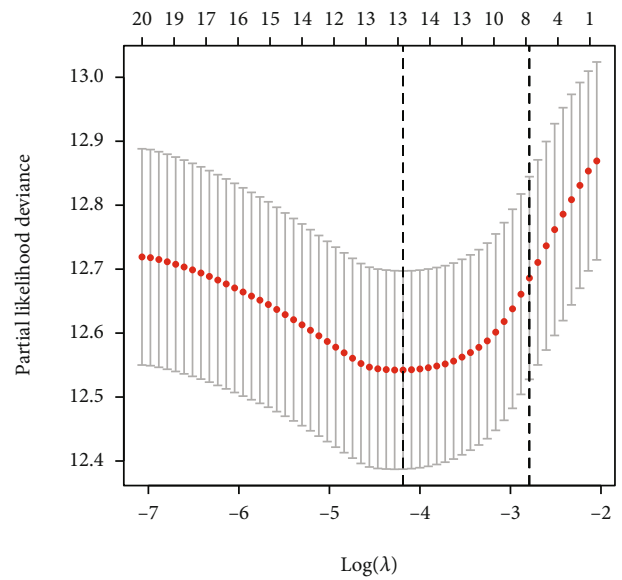

(e)

FIGURE 6: Drug sensitivity analysis and signaling pathway analysis of the glycolysis-related genes and the process of establishing the risk signature. (a) Red indicates that the gene is positively correlated with drug sensitivity. Purple represents that the gene is negatively correlated with drug sensitivity. (b) Univariate Cox regression analysis. (c) Activation or inhibition of different signaling pathways by glycolysis-related genes. (d), (e) LASSO regression analysis and verification.

increased expression of ENO2 promotes glycolysis in gastric cancer cells, which is related to tumor growth and liver metastasis [40]. In terms of Warburg effect in renal cell carcinoma, recent studies proposed that the expression of ENO2 was significantly higher in the tissues and serum of RCC patients [41, 42]. In addition, the increase in serum ENO2 level was related to clinical stage, tumor grade, and disease recurrence; therefore, it is a potential biomarker for the prognosis of RCC [43, 44]. In our study, ENO2 was highly expressed in the tissues of ccRCC patients and played a role in activating the cell cycle, EMT, and PI3K/AKT signaling pathway, which was consistent with our expectations. Meanwhile, the results indicated that $\mathrm{ENO} 2$ may promote the growth and invasion of ccRCC cells through glycolysis and 


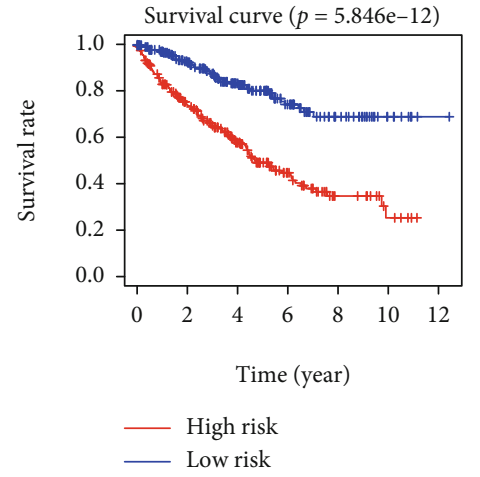

(a)

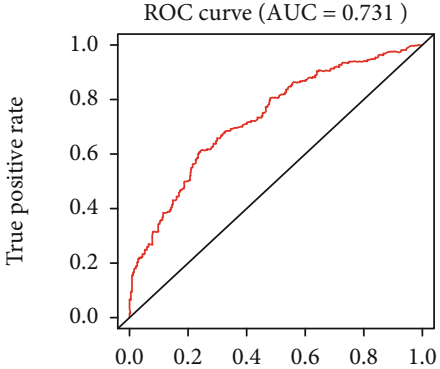

False positive rate

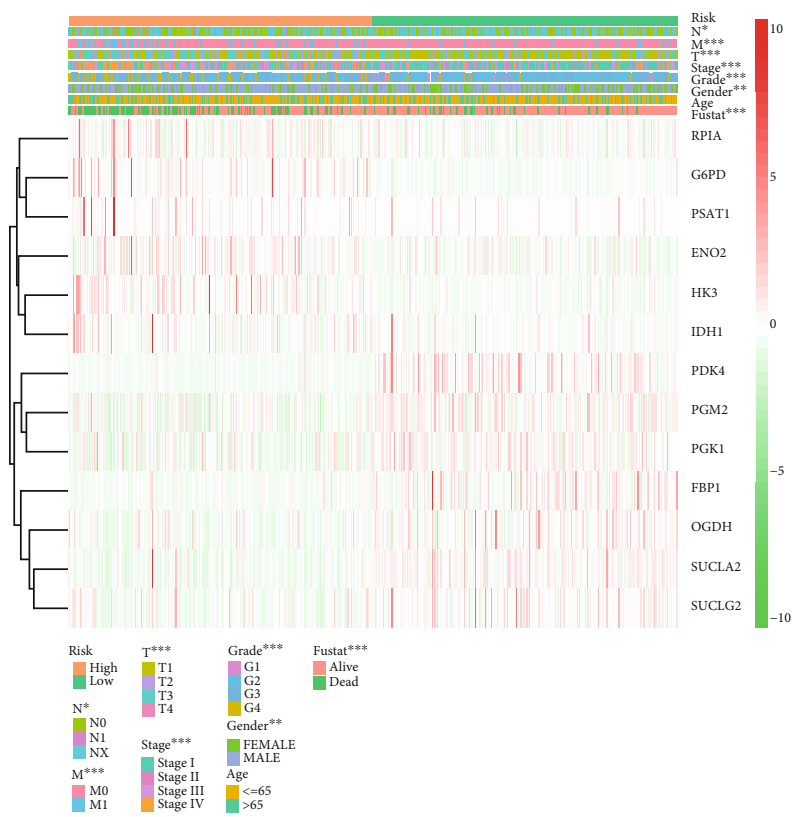

(c)

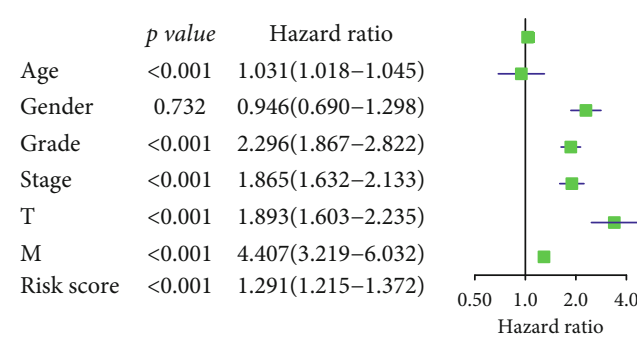

(d)

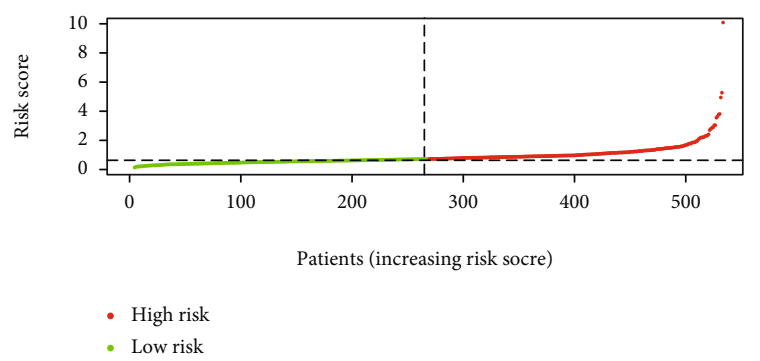

(f)

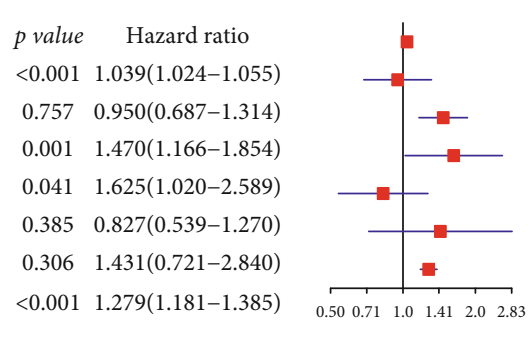

Hazard ratio

(e)

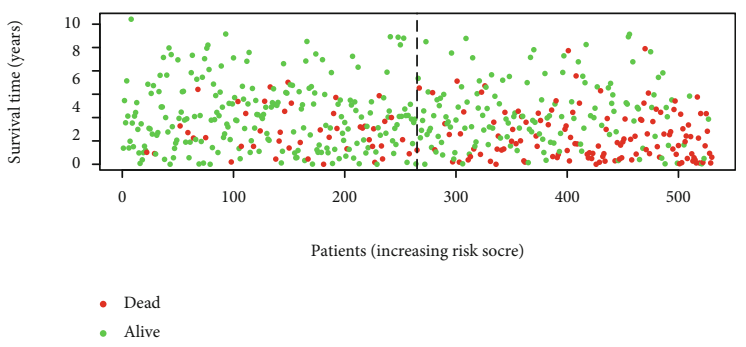

(g)

Figure 7: Continued. 


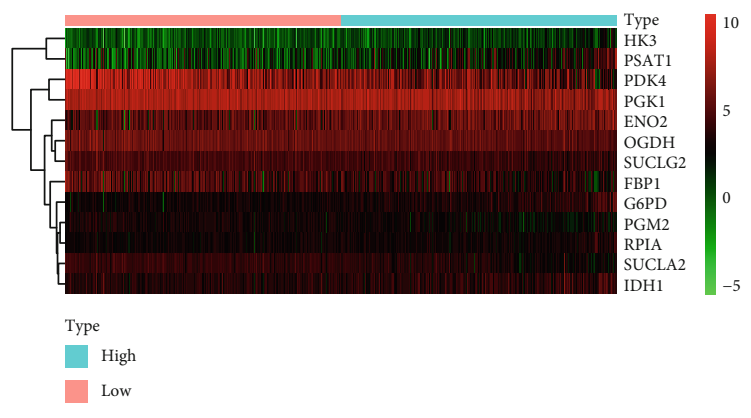

(h)

FIGURE 7: Relationship between the risk scores, clinicopathological factors, and clusters. (a) Kaplan-Meier overall survival (OS) curves based on this risk model. (b) ROC curves showed the predictive ability of the risk signature. (c) The heat map showed the expression levels of the 13 glycolysis-related genes in low- and high-risk ccRCC patients and the correlation analysis with clinicopathological factors. (d) Univariate Cox regression analyses. (e) Multivariate Cox regression analyses. (f) The relationship between risk scores and high risk and low risk. (g) The relationship between risk scores and survival time. (h) The heat map showed the expression levels of the 13 glycolysis-related genes in low- and high-risk ccRCC patients.

that the high expression of ENO2 may be related to the Warburg effect.

Metabolic transfer to glycolysis is related to changes in the signaling pathways involved in energy metabolism. This process involves the ingestion and fermentation of glucose. HK3 is a key gene in glycolysis, and the first step of hexokinase- (HK-) catalyzed glycolysis has been confirmed to be very important in the development of colorectal cancer and melanoma [45]. The expression of glycolytic enzymes, including $\mathrm{HK} 3$, was also increased in breast cancer, and HK3 was considered to be the most important gene in pediatric acute lymphoblastic leukemia; it plays an important role in the prognosis of the disease through the glycolytic pathway $[46,47]$. The increased expression of HK3 is related to EMT in colorectal cancer, which is involved in the rapid growth and metastasis of colorectal cancer [48]. At present, there are few reports on $\mathrm{HK} 3$ in renal cell carcinoma. In our study, HK3 played a role in activating apoptosis and the EMT signaling pathway, which is consistent with previous research results. Based on the expression of $\mathrm{HK} 3$ in other tumors, we speculated that the high expression of HK3 in ccRCC was related to the Warburg effect, and HK3 could promote the rapid proliferation, invasion, and metastasis of ccRCC by enhancing apoptosis and epithelial-mesenchymal transition.

FBP1 catalyzes the formation of fructose-6-phosphate from fructose-1,6-diphosphate and water, which plays a key role in gluconeogenesis. FBP1 is a downstream glycoisoenzyme and tumor suppressor that inhibits glycolysis and tumor growth and partially inhibits tumor growth by inhibiting mitotic signal transduction $[49,50]$. As a tumor suppressor, decreased expression of FBPT1 in a variety of tumors has been reported in many studies. The low expression of FBP1 promoted the invasion of hepatocellular carcinoma cells through the Warburg effect [51]. When FBP1 was downregulated, glycolysis increased, and the decrease in FBP1 level reprogrammed the metabolism of glioblastoma cells [52]. The expression of FBP1 was downregulated in gastric cancer and gastric cancer cell lines, and this downregulation was related to the Warburg effect in tumors [53]. High expression of FBP1 inhibits the growth, metastasis, and glycolysis of breast cancer [54]. Previous studies have shown that FBP1 could inhibit glycolysis in kidney cancer, and the expression of FBP1was significantly lower in patients with high-grade ccRCC than in patients with low-grade ccRCC [55]. FBP1 could antagonize the glycolytic flux of renal tubular epithelial cells, the assumed cells of ccRCC, thus inhibiting the Warburg effect [56]. In our study, FBP1 was downregulated in the tissues of ccRCC patients, suggesting that glycolysis was enhanced in ccRCC, while gluconeogenesis was decreased, which was consistent with our expectations. In addition, FBP1 plays a role in inhibiting the cell cycle and EMT pathways, and thus, FBP1 had an inhibitory effect on tumor growth, invasion, and metastasis.

These genes are closely related to the pathological characteristics of cancer. According to clinical characteristics, ccRCC patients were divided into the high-risk and lowrisk groups. Compared with the low-risk group patients, the high-risk group patients had a higher proportion of RPIA, G6PD, PSAT1, ENO2, HK3, IDH1, and lower proportions of PDK4, PGM2, PGK1, FBP1, OGDH, SUCLA2, and SUCLG2. As the risk score, age, stage, grade, $\mathrm{T}$ status, and $M$ status increased, the risk increased. The Kaplan-Meier overall survival curves showed that the high-risk group had a lower survival rate than the low-risk group, which suggested that the predictive model could predict the survival rate and evaluate the prognosis of ccRCC patients.

The results of drug sensitivity analysis suggested that the low expression of ENO2 and HK3 genes and the high expression of PGK1 were positively correlated with drug sensitivity. In addition, some glycolysis-related genes were positively correlated with drug sensitivity, which could be used as potential targets for drug therapy. We performed a transcription factor network for the main differentially expressed genes of ccRCC, and several core transcriptional factors were identified: FLI1, ETS1, SREBF2, PML, CEBPB, RUNX1, MYBL2, CENPA, FOXM1, and LMNB1. The discovery of these transcription factors provides a basis for protein and functional research.

In this study, we first explored the expression of glycolysis-related genes in ccRCC using integrated 
bioinformatic analysis. We identified key glycolysis-related genes that are differentially expressed in ccRCC, analyzed the effects of these genes on common signaling pathways, and generated a predictive prognostic model for ccRCC. However, there are still some limitations. Our results have not been validated by in vitro experiments, such as quantitative real-time polymerase chain reaction and western blotting. Further studies on human tissue samples are required to validate these results.

\section{Conclusions}

In conclusion, we identified that key glycolysis-related genes are differentially expressed in ccRCC, analyzed the effects of these genes on common signaling pathways, and constructed a predictive model of ccRCC. This predictive model correlated well with the development and progression of ccRCC and showed great diagnostic and prognostic value. This model may serve as a potential prognostic biomarker and drug target for ccRCC.

\section{Abbreviations}

CNV: $\quad$ Copy number variations

SNV: $\quad$ Single nucleotide variant

ccRCC: Clear cell renal cell carcinoma

TCGA: The Cancer Genome Atlas

PSAT1: Phosphoserine aminotransferase 1

SDHB: Succinate dehydrogenase complex iron sulfur subunit B

RPIA: Ribose 5-phosphate isomerase A

G6PD: Glucose-6-phosphate dehydrogenase

ENO2: Enolase 2

HK3: Hexokinase 3

IDH1: Isocitrate dehydrogenase 1

PDK4: $\quad$ Pyruvate dehydrogenase kinase 4

PGM2: $\quad$ Phosphoglucomutase 2

PGK1: $\quad$ Phosphoglycerate kinase 1

FBP1: $\quad$ Fructose-bisphosphatase 1

OGDH: Oxoglutarate dehydrogenase

SUCLA2: Succinate-CoA ligase ADP-forming subunit beta

SUCLG2: Succinate-CoA ligase GDP-forming subunit beta

GEPIA: Gene expression profiling interactive analysis

OS: $\quad$ Overall survival

DFS: Disease-free survival.

\section{Data Availability}

The data used to support the findings of this study are available from the corresponding author upon request.

\section{Conflicts of Interest}

No authors declared any potential conflicts of interest.

\section{Authors' Contributions}

Yan Zhang and Mingying Chen contributed equally to this study. Yan Zhang and Mingying Chen are the co-first authors. Guangzhen $\mathrm{Wu}$ and Yingkun $\mathrm{Xu}$ designed the study and collected the data. Yan Zhang and Mingying Chen performed the data analysis and wrote the manuscript. Guangzhen $\mathrm{Wu}$, Yingkun $\mathrm{Xu}$, and Meihong Liu revised the manuscript.

\section{Acknowledgments}

We thank The Cancer Genome Atlas (TCGA) for providing the publicly available data. This project was supported by the Liaoning Provincial Department of Education's 2020 scientific research project (No. LZ2020071). In addition, Yingkun $\mathrm{Xu}$ would also like to thank Jiayi Li for useful discussions regarding this research.

\section{References}

[1] J. Oto, E. Plana, J. V. Sánchez-González et al., "Urinary microRNAs: looking for a new tool in diagnosis, prognosis, and monitoring of renal cancer," Current Urology Reports, vol. 21, no. 2, p. 11, 2020.

[2] T. Xu, H. Ruan, Z. Song et al., "Identification of CXCL13 as a potential biomarker in clear cell renal cell carcinoma via comprehensive bioinformatics analysis," Biomedicine \& Pharmacotherapy, vol. 118, p. 109264, 2019.

[3] F. Bray, J. Ferlay, I. Soerjomataram, R. L. Siegel, L. A. Torre, and A. Jemal, "Global cancer statistics 2018: GLOBOCAN estimates of incidence and mortality worldwide for 36 cancers in 185 countries," CA: a Cancer Journal for Clinicians, vol. 68, no. 6, pp. 394-424, 2018.

[4] R. L. Siegel, K. D. Miller, and A. Jemal, "Cancer statistics, 2019," CA: a Cancer Journal for Clinicians, vol. 69, no. 1, pp. 7-34, 2019.

[5] A. Bokhari and P. G. Tiscornia-Wasserman, "Cytology diagnosis of metastatic clear cell renal cell carcinoma, synchronous to pancreas, and metachronous to thyroid and contralateral adrenal: report of a case and literature review," Diagnostic Cytopathology, vol. 45, no. 2, pp. 161-167, 2017.

[6] M. Wang, Y. Sun, J. Xu et al., "Preclinical studies using miR32-5p to suppress clear cell renal cell carcinoma metastasis via altering the miR-32-5p/TR4/HGF/Met signaling," International Journal of Cancer, vol. 143, no. 1, pp. 100-112, 2018.

[7] P. Vaupel, H. Schmidberger, and A. Mayer, "The Warburg effect: essential part of metabolic reprogramming and central contributor to cancer progression," International Journal of Radiation Biology, vol. 95, no. 7, pp. 912-919, 2019.

[8] E. Racker, "Why do tumor cells have a high aerobic glycolysis?," Journal of Cellular Physiology, vol. 89, no. 4, pp. 697700, 1976.

[9] Y. Kobayashi, K. Banno, H. Kunitomi et al., "Warburg effect in gynecologic cancers," The Journal of Obstetrics and Gynaecology Research, vol. 45, no. 3, pp. 542-548, 2019.

[10] G. Wu, Q. Wang, Y. Xu et al., "Targeting the transcription factor receptor LXR to treat clear cell renal cell carcinoma: agonist or inverse agonist?," Cell Death \& Disease, vol. 10, no. 6, p. 416, 2019.

[11] Y. Guo, F. Liang, F. Zhao, and J. Zhao, "Resibufogenin suppresses tumor growth and Warburg effect through regulating miR-143-3p/HK2 axis in breast cancer," Molecular and Cellular Biochemistry, vol. 466, no. 1-2, pp. 103-115, 2020.

[12] Y. Zhou, X. Zheng, J. Lu, W. Chen, X. Li, and L. Zhao, "Ginsenoside 20(S)-Rg3 inhibits the Warburg effect via modulating DNMT3A/ MiR-532-3p/HK2 pathway in ovarian cancer 
cells," Cellular Physiology and Biochemistry, vol. 45, no. 6, pp. 2548-2559, 2018.

[13] S. Han, S. Yang, Z. Cai et al., "Anti-Warburg effect of rosmarinic acid via miR-155 in gastric cancer cells," Drug Design, Development and Therapy, vol. 9, pp. 2695-2703, 2015.

[14] J. Zheng, J. Luo, H. Zeng, L. Guo, and G. Shao, “125 I suppressed the Warburg effect_via_ regulating miR-338/PFKL axis in hepatocellular carcinoma," Biomedicine \& Pharmacotherapy, vol. 119, p. 109402, 2019.

[15] W. Peng, W. Huang, X. Ge, L. Xue, W. Zhao, and J. Xue, “Type I $\gamma$ phosphatidylinositol phosphate kinase promotes tumor growth by facilitating Warburg effect in colorectal cancer," eBioMedicine, vol. 44, pp. 375-386, 2019.

[16] L. Liu, J. Cao, J. Zhao, X. Li, Z. Suo, and H. Li, "PDHA1 gene knockout in human esophageal squamous cancer cells resulted in greater Warburg effect and aggressive features in vitro and in vivo," Oncotargets and Therapy, vol. Volume 12, pp. 9899-9913, 2019.

[17] Y. Liu, T. Liang, X. Qiu et al., "Down-regulation of Nfatc1 suppresses proliferation, migration, invasion, and Warburg effect in prostate cancer cells," Medical Science Monitor, vol. 25, pp. 1572-1581, 2019.

[18] G. Zhang, J. Li, X. Wang et al., "The reverse Warburg effect and 18F-FDG uptake in non-small cell lung cancer A549 in mice: a pilot study," Journal of Nuclear Medicine, vol. 56, no. 4, pp. 607-612, 2015.

[19] T. Takai, Y. Yoshikawa, T. Inamoto et al., “A novel combination RNAi toward Warburg effect by replacement with miR145 and silencing of PTBP1 induces apoptotic cell death in bladder cancer cells," International Journal of Molecular Sciences, vol. 18, no. 1, p. 179, 2017.

[20] D. S. Chandrashekar, B. Bashel, S. A. H. Balasubramanya et al., "UALCAN: a portal for facilitating tumor subgroup gene expression and survival analyses," Neoplasia, vol. 19, no. 8, pp. 649-658, 2017.

[21] A. A. Hakimi, E. Reznik, C. H. Lee et al., "An integrated metabolic atlas of clear cell renal cell carcinoma," Cancer Cell, vol. 29, no. 1, pp. 104-116, 2016.

[22] C. J. Liu, F. F. Hu, M. X. Xia, L. Han, Q. Zhang, and A. Y. Guo, "GSCALite: a web server for gene set cancer analysis," Bioinformatics, vol. 34, no. 21, pp. 3771-3772, 2018.

[23] Z. Tang, C. Li, B. Kang, G. Gao, C. Li, and Z. Zhang, "GEPIA: a web server for cancer and normal gene expression profiling and interactive analyses," Nucleic Acids Research, vol. 45, no. W1, pp. W98-w102, 2017.

[24] P. Shannon, A. Markiel, O. Ozier et al., "Cytoscape: a software environment for integrated models of biomolecular interaction networks," Genome Research, vol. 13, no. 11, pp. 24982504, 2003.

[25] W. Yang, J. Soares, P. Greninger et al., "Genomics of Drug Sensitivity in Cancer (GDSC): a resource for therapeutic biomarker discovery in cancer cells," Nucleic Acids Research, vol. 41, no. Database issue, pp. D955-D961, 2013.

[26] V. A. Kobliakov, "The mechanisms of regulation of aerobic glycolysis (Warburg effect) by oncoproteins in carcinogenesis," Biochemistry, vol. 84, no. 10, pp. 1117-1128, 2019.

[27] H. Geng, C. Xue, J. Mendonca et al., "Interplay between hypoxia and androgen controls a metabolic switch conferring resistance to androgen/AR-targeted therapy," Nat Commun, vol. 9, no. 1, p. 4972, 2018.
[28] R. Kelsey, "Glycolysis and AR expression as biomarkers," Nature Reviews Urology, vol. 15, no. 1, p. 2, 2018.

[29] R. Hoefflin, S. Harlander, S. Schäfer et al., "HIF- $1 \alpha$ and HIF- $2 \alpha$ differently regulate tumour development and inflammation of clear cell renal cell carcinoma in mice," Nature Communications, vol. 11, no. 1, p. 4111, 2020.

[30] Z. Abbaszadeh, S. Çeşmeli, and Ç. Biray Avcı, "Crucial players in glycolysis: cancer progress," Gene, vol. 726, article 144158, 2020.

[31] M. Morais, F. Dias, A. L. Teixeira, and R. Medeiros, "MicroRNAs and altered metabolism of clear cell renal cell carcinoma: potential role as aerobic glycolysis biomarkers," Biochimica et Biophysica Acta - General Subjects, vol. 1861, no. 9, pp. 2175-2185, 2017.

[32] Y. Xu, G. Wu, J. Zhang et al., "TRIM33 overexpression inhibits the progression of clear cell renal cell carcinoma in vivo and in vitro," BioMed Research International, vol. 2020, Article ID 8409239, 18 pages, 2020.

[33] J. Li, G. Wu, Y. Xu et al., "Porcupine inhibitor LGK974 downregulates the Wnt signaling pathway and inhibits clear cell renal cell carcinoma," BioMed Research International, vol. 2020, Article ID 2527643, 16 pages, 2020.

[34] J. Elliott, B. Bodinier, T. A. Bond et al., "Predictive accuracy of a polygenic risk score-enhanced prediction model vs a clinical risk score for coronary artery disease," JAMA, vol. 323, no. 7, pp. 636-645, 2020.

[35] Y. Xu, X. Li, Y. Han et al., "A new prognostic risk model based on PPAR pathway-related genes in kidney renal clear cell carcinoma," PPAR Research, vol. 2020, Article ID 6937475, 13 pages, 2020.

[36] P. Pal Choudhury, A. N. Wilcox, M. N. Brook et al., "Comparative validation of breast cancer risk prediction models and projections for future risk stratification," Journal of the National Cancer Institute, vol. 112, no. 3, pp. 278-285, 2020.

[37] C. C. Liu, H. Wang, W. D. Wang et al., "ENO2 promotes cell proliferation, glycolysis, and glucocorticoid-resistance in acute lymphoblastic leukemia," Cellular Physiology and Biochemistry, vol. 46, no. 4, pp. 1525-1535, 2018.

[38] M. G. Kounelakis, M. E. Zervakis, G. C. Giakos, G. J. Postma, L. M. C. Buydens, and X. Kotsiakis, "On the relevance of glycolysis process on brain gliomas," IEEE Journal of Biomedical and Health Informatics, vol. 17, no. 1, pp. 128-135, 2013.

[39] C. S. Yeh, J. Y. Wang, F. Y. Chung et al., "Significance of the glycolytic pathway and glycolysis related-genes in tumorigenesis of human colorectal cancers," Oncology Reports, vol. 19, no. 1, pp. 81-91, 2008.

[40] Q. Wang, C. Chen, Q. Ding et al., "METTL3-mediated m6A modification of HDGF mRNA promotes gastric cancer progression and has prognostic significance," Gut, vol. 69, no. 7, pp. 1193-1205, 2020.

[41] E. Sanders and S. Diehl, "Analysis and interpretation of transcriptomic data obtained from extended Warburg effect genes in patients with clear cell renal cell carcinoma," Oncoscience, vol. 2, no. 2, pp. 151-186, 2015.

[42] P. N. Teng, B. L. Hood, M. Sun, R. Dhir, and T. P. Conrads, "Differential proteomic analysis of renal cell carcinoma tissue interstitial fluid," Journal of Proteome Research, vol. 10, no. 3, pp. 1333-1342, 2011.

[43] T. Rasmuson, K. Grankvist, and B. Ljungberg, "Serum gammaenolase and prognosis of patients with renal cell carcinoma," Cancer, vol. 72, no. 4, pp. 1324-1328, 1993. 
[44] M. Takashi, T. Sakata, and K. Kato, "Use of serum gammaenolase and aldolase A in combination as markers for renal cell carcinoma," Japanese Journal of Cancer Research, vol. 84, no. 3, pp. 304-309, 1993.

[45] A. V. Kudryavtseva, M. S. Fedorova, A. Zhavoronkov et al., "Effect of lentivirus-mediated shRNA inactivation of HK1, $\mathrm{HK} 2$, and HK3 genes in colorectal cancer and melanoma cells," BMC Genetics, vol. 17, no. S3, p. 156, 2016.

[46] H. Harami-Papp, L. S. Pongor, G. Munkácsy et al., “TP53 mutation hits energy metabolism and increases glycolysis in breast cancer," Oncotarget, vol. 7, no. 41, pp. 67183-67195, 2016.

[47] H. Y. Gao, X. G. Luo, X. Chen, and J. H. Wang, "Identification of key genes affecting disease free survival time of pediatric acute lymphoblastic leukemia based on bioinformatic analysis," Blood Cells, Molecules \& Diseases, vol. 54, no. 1, pp. 3843, 2015.

[48] E. A. Pudova, A. V. Kudryavtseva, M. S. Fedorova et al., "HK3 overexpression associated with epithelial-mesenchymal transition in colorectal cancer," BMC Genomics, vol. 19, no. S3, p. 113, 2018.

[49] G. Grasmann, E. Smolle, H. Olschewski, and K. Leithner, "Gluconeogenesis in cancer cells - repurposing of a starvation-induced metabolic pathway?," Biochimica Et Biophysica Acta. Reviews on Cancer, vol. 1872, no. 1, pp. 24-36, 2019.

[50] M. T. Snaebjornsson and A. Schulze, "Non-canonical functions of enzymes facilitate cross-talk between cell metabolic and regulatory pathways," Experimental \& Molecular Medicine, vol. 50, no. 4, p. 34, 2018.

[51] J. Yang, C. Wang, F. Zhao et al., "Loss of FBP1 facilitates aggressive features of hepatocellular carcinoma cells through the Warburg effect," Carcinogenesis, vol. 38, no. 2, pp. 134143, 2017.

[52] B. Son, S. Lee, H. Kim et al., "Decreased FBP1 expression rewires metabolic processes affecting aggressiveness of glioblastoma," Oncogene, vol. 39, no. 1, pp. 36-49, 2020.

[53] X. Liu, X. Wang, J. Zhang et al., "Warburg effect revisited: an epigenetic link between glycolysis and gastric carcinogenesis," Oncogene, vol. 29, no. 3, pp. 442-450, 2010.

[54] L. Shi, C. He, Z. Li, Z. Wang, and Q. Zhang, "FBP1 modulates cell metabolism of breast cancer cells by inhibiting the expression of HIF-1 $\alpha$," Neoplasma, vol. 64, no. 4, pp. 535-542, 2017.

[55] K. Leithner, "Epigenetic marks repressing gluconeogenesis in liver and kidney cancer," Cancer Research, vol. 80, no. 4, pp. 657-658, 2020.

[56] B. Li, B. Qiu, D. S. M. Lee et al., "Fructose-1,6-bisphosphatase opposes renal carcinoma progression," Nature, vol. 513, no. 7517, pp. 251-255, 2014. 